\title{
A SINGULAR PERTURBATION PROBLEM IN A SYSTEM OF NONLINEAR SCHRÖDINGER EQUATION OCCURRING IN LANGMUIR TURBULENCE
}

\author{
CÉDric Galusinski ${ }^{1}$
}

\begin{abstract}
The aim of this work is to establish, from a mathematical point of view, the limit $\alpha \rightarrow+\infty$ in the system

$$
i \partial_{t} E+\nabla(\nabla \cdot E)-\alpha^{2} \nabla \times \nabla \times E=-|E|^{2 \sigma} E,
$$

where $E: \mathbb{R}^{3} \rightarrow \mathbb{C}^{3}$. This corresponds to an approximation which is made in the context of Langmuir turbulence in plasma Physics. The $L^{2}$-subcritical $\sigma$ (that is $\sigma \leq 2 / 3$ ) and the $H^{1}$-subcritical $\sigma$ (that is $\sigma \leq 2)$ are studied. In the physical case $\sigma=1$, the limit is then studied for the $H^{1}\left(\mathbb{R}^{3}\right)$ norm.
\end{abstract}

Mathematics Subject Classification. 35B05, 35B25, 35L60.

Received: November 12, 1998. Revised: July 23, 1999.

\section{Setting of the problem and main Results}

We are interested in the first Schrödinger system that describes the coupled motion of intermingled electrons and ion fluids that compose the plasma without any magnetic field. The system satisfied by the electric field envelope $\bar{E}$ is

$$
-2 i \omega_{\mathrm{pe}} \partial_{t} \bar{E}-3 v_{\mathrm{Te}}^{2} \nabla(\nabla \cdot \bar{E})+c^{2} \nabla \times \nabla \times \bar{E}+\omega_{\mathrm{pe}}{ }^{2} \frac{\delta n}{n_{0}} \bar{E}=0
$$

where $\omega_{\text {pe }}$ is the electron plasma frequency, $v_{\mathrm{Te}}$ the average thermal velocity of the electrons, $c$ the velocity of light, $n_{0}$ the mean density of electrons and $\delta n_{\mathrm{e}}$ is the low frequency variation of electron density. A justification for the time-envelope approximation can be found in [1].

Writing $\frac{\delta n}{n_{0}}$ in terms of $\bar{E}$ in (1.1), we obtain

$$
i \omega_{\mathrm{pe}} \partial_{t} \bar{E}+\frac{3}{2} v_{\mathrm{Te}}^{2} \nabla(\nabla \cdot \bar{E})-\frac{c^{2}}{2} \nabla \times \nabla \times \bar{E}+\frac{\varepsilon_{0} \omega_{\mathrm{pe}}^{2}}{8 n_{0} k_{B} T_{\mathrm{e}}}|\bar{E}|^{2} \bar{E}=0
$$

where $\varepsilon_{0}$ is the dielectric permittivity of free space, $k_{B}$ the Boltzmann's constant, and $k_{B} T_{\mathrm{e}}$ is the electron thermal energy.

Keywords and phrases. Nonlinear Schrödinger equation, singular perturbation.

1 Université Bordeaux I, Mathématiques Appliquées Bordeaux, ESA 5466 CNRS, 351 cours de la libération, 33400 Talence, France. e-mail: galusins@math.u-bordeaux.fr 
The term $\frac{c^{2}}{2} \nabla \times \nabla \times \bar{E}$ is usually neglected in physics, the equation (1.2) becomes (see [3], p. 138),

$$
i \omega_{\mathrm{pe}} \partial_{t} \bar{E}+\frac{3}{2} v_{\mathrm{Te}}^{2} \nabla(\nabla \cdot \bar{E})+\frac{\varepsilon_{0} \omega_{\mathrm{pe}}^{2}}{8 n_{0} k_{B} T_{\mathrm{e}}}|\bar{E}|^{2} \bar{E}=0
$$

Equations that have the general form of (1.3) widely occur in physics and are generically known as the nonlinear Schrödinger equation. The reader can refer to [3] for more details on the physical problem.

The goal of this paper is to verify, from a mathematical point of view, if the term $\frac{c^{2}}{2} \nabla \times \nabla \times \bar{E}$ can be simply neglected in (1.2) or not. Since $v_{\mathrm{Te}}^{2} \ll c^{2}$, the following model is considered

$$
\begin{gathered}
i E_{t}+\nabla \nabla \cdot E-\alpha^{2} \nabla \times \nabla \times E=\varepsilon|E|^{2 \sigma} E, \\
E_{\mid t=0}=E_{0},
\end{gathered}
$$

with $\alpha>1$ and we investigate the case $\alpha \rightarrow \infty$.

The parameter $\varepsilon$ takes the value 1 or -1 . The complex variable $E$ depends on $t \in \mathbb{R}$ and $x \in \mathbb{R}^{3}$.

Rather than studying this problem for $x \in \mathbb{R}^{3}$, we generalize this work to the case $x \in \mathbb{R}^{N}$ for $N \geq 2$. We then replace (1.4) by

$$
\begin{gathered}
i E_{\alpha t}+\nabla \nabla \cdot E_{\alpha}+\alpha^{2}(\triangle-\nabla \nabla .) E_{\alpha}=\varepsilon\left|E_{\alpha}\right|^{2 \sigma} E_{\alpha} \text { in } \mathcal{D}^{\prime}\left(\mathbb{R}^{N}\right), \\
E_{\alpha \mid t=0}=E_{0} .
\end{gathered}
$$

The following splitting will be useful in the sequel,

$$
\begin{gathered}
i E_{\|_{t}}+\triangle E_{\|}=\varepsilon \mathcal{P}_{\|}\left(\left|E_{\|}+E_{\perp}\right|^{2 \sigma}\left(E_{\|}+E_{\perp}\right)\right) \text { in } \mathcal{D}^{\prime}\left(\mathbb{R}^{N}\right), \\
E_{\| t=0}=\mathcal{P}_{\|} E_{0} \in L^{2}\left(\mathbb{R}^{N}\right), \\
i E_{\perp t}+\alpha^{2} \triangle E_{\perp}=\varepsilon \mathcal{P}_{\perp}\left(\left|E_{\|}+E_{\perp}\right|^{2 \sigma}\left(E_{\|}+E_{\perp}\right)\right) \text { in } \mathcal{D}^{\prime}\left(\mathbb{R}^{N}\right), \\
E_{\perp \mid t=0}=\mathcal{P}_{\perp} E_{0} \in L^{2}\left(\mathbb{R}^{N}\right),
\end{gathered}
$$

where $\mathcal{P}_{\perp}$ denotes the Hodge projection from $L^{2}\left(\mathbb{R}^{N}\right)$ to the subspace of divergence free vector fields, $\mathcal{P}_{\|}=$ $I-\mathcal{P}_{\perp}, E_{\|}=\mathcal{P}_{\|} E_{\alpha}$ and $E_{\perp}=\mathcal{P}_{\perp} E_{\alpha}$.

The results about existence and uniqueness of a solution for (1.5) are similar to the ones concerning nonlinear Schrödinger equations $[2,4,5]$. The first step is the study of the linear problem in order to obtain uniform (with respect to $\alpha$ ) estimates on $E_{\|}$and $E_{\perp}$, defined in (1.6), (1.7). The term $E_{\perp}$ being small in some sense for large value of $\alpha$, the limit of the solution of (1.5) can be evaluated.

The limit obtained here reads:

$$
\begin{aligned}
i E_{t}+\triangle E & =\varepsilon \mathcal{P}_{\|}\left(|E|^{2 \sigma} E\right) \\
E_{\mid t=0} & =E_{0 \|}
\end{aligned}
$$

and $E=\mathcal{P}_{\|} E$. Remark that here, $\triangle E=\nabla \nabla . E$.

Remark also that the equation (1.8) is quite different of (1.3) which corresponds to

$$
i E_{t}+\nabla \nabla \cdot E=\varepsilon|E|^{2 \sigma} E .
$$

In what follows, we establish the convergence of solutions of (1.5) to the ones of (1.8) for adequate norms.

Let us recall from [2] some results on (1.8). 
Theorem 1.1. Let $E_{0} \in L^{2}\left(\mathbb{R}^{N}\right)$ and $\sigma<\frac{2}{N}$; there exists an unique solution $E \in \mathcal{C}\left(\mathbb{R}^{+} ; L^{2}\left(\mathbb{R}^{N}\right)\right) \cap L_{\text {loc }}^{q}([0,+\infty[$; $\left.L^{2 \sigma+2}\left(\mathbb{R}^{N}\right)\right)$ to $(1.8)$, where $\frac{2}{q}=\frac{N}{2}-\frac{N}{2 \sigma+2}$.

Let $E_{0} \in H^{1}\left(\mathbb{R}^{N}\right)$ and $\sigma<\frac{2}{N-2}$; there exist $T^{*}>0$ and a unique maximal solution $E \in \mathcal{C}\left(\mathbb{R}^{+} ; H^{1}\left(\mathbb{R}^{N}\right)\right)$. There exist some initial data $E_{0} \in H^{1}\left(\mathbb{R}^{N}\right)$ (when $\varepsilon=-1$ and $\sigma>\frac{2}{N}$ ) for which $T^{*}$ is finite.

The main results consist of the next Theorems whose proofs are developed in Section 4.

Theorem 1.2. Assume $\sigma<\frac{2}{N}$. Let $E_{0} \in L^{2}\left(\mathbb{R}^{N}\right)$, there exists a unique global solution $E_{\alpha} \in \mathcal{C}\left(\mathbb{R}^{+} ; L^{2}\left(\mathbb{R}^{N}\right)\right) \cap$ $L_{\text {loc }}^{q}\left(\left[0,+\infty\left[; L^{2 \sigma+2}\left(\mathbb{R}^{N}\right)\right)\right.\right.$ (where $\left.\frac{2}{q}=\frac{N}{2}-\frac{N}{2 \sigma+2}\right)$ to $(1.5)$.

Moreover,

$$
\begin{gathered}
E_{\alpha} \rightarrow E \text { in } L^{q}\left(\left[0, T\left[; L^{2 \sigma+2}\left(\mathbb{R}^{N}\right)\right) \text { strongly }\left(\frac{2}{q}=\frac{N}{2}-\frac{N}{2 \sigma+2}\right)\right.\right. \\
E_{\alpha}(t)-e^{-i \alpha^{2} \triangle t} E_{0 \perp} \rightarrow E \text { in } L^{\infty}\left(\left[0, T\left[; L^{2}\left(\mathbb{R}^{N}\right)\right) \forall T<+\infty\right.\right.
\end{gathered}
$$

where $E$ is the solution to (1.8) introduced in Theorem 1.1.

Theorem 1.3. Assume $\sigma<\frac{2}{N-2}$. Let $E_{0} \in H^{1}\left(\mathbb{R}^{N}\right)$. Then there exist $T_{\alpha}>0$ and a unique maximal solution $E_{\alpha} \in \mathcal{C}\left(\left[0, T_{\alpha}\left[; H^{1}\left(\mathbb{R}^{N}\right)\right)\right.\right.$ to (1.5). Moreover $\liminf _{\alpha \rightarrow+\infty} T_{\alpha} \geq T^{*}$ where $T^{*}$ is the existence time of the solution $E$ to (1.8) introduced in theorem 1.1 (if $\varepsilon=1$ or if $\varepsilon=-1$ and $\sigma<\frac{2}{N}$ then $T_{\alpha}=T^{*}=+\infty$ ). If $\mathcal{P}_{\perp} E_{0} \rightarrow 0$ in $H^{1}\left(\mathbb{R}^{N}\right)$ when $\alpha$ goes to infinity, then,

$$
\begin{gathered}
E_{\alpha} \rightarrow E \text { in } W^{1, q}\left(\left[0, T\left[; L^{2 \sigma+2}\left(\mathbb{R}^{N}\right)\right) \text { strongly }\left(\frac{2}{q}=\frac{N}{2}-\frac{N}{2 \sigma+2}\right)\right.\right. \\
E_{\alpha}(t) \rightarrow E \text { in } \mathcal{C}\left(\left[0, T\left[; H^{1}\left(\mathbb{R}^{N}\right)\right) \forall T<T^{*}\right.\right.
\end{gathered}
$$

Remark on notation. Throughout this article, the same letter $C$ (or sometimes $c$ ) shall denote constants which may change from line to line.

The classical space $L^{p}\left(\mathbb{R}^{N}\right)$ is sometimes noted $L^{p}$ for conciseness. Some notations will be introduced in the Preliminaries.

\section{Preliminaries}

\subsection{The linear group}

In this section, we study the following linear groups, $S_{\|}(t)$ associated to

$$
\begin{gathered}
i E_{\|_{t}}+\nabla \nabla \cdot E_{\|}=0 \\
E_{\|_{\mid t=0}}=E_{0 \|} \in L^{2}\left(\mathbb{R}^{N}\right),
\end{gathered}
$$

and $S_{\perp}(t)$ associated to

$$
\begin{gathered}
i E_{\perp t}+\alpha^{2}(\triangle-\nabla \nabla .) E_{\perp}=0 \\
E_{\perp \mid t=0}=E_{0 \perp} \in L^{2}\left(\mathbb{R}^{N}\right) .
\end{gathered}
$$

Simple computations with Fourier transform lead to

$$
\begin{gathered}
\widehat{E_{\|}}(t)=e^{-i|\xi|^{2} t} M \widehat{E_{0 \|}}, \\
\widehat{E_{\perp}}(t)=e^{-i \alpha^{2}|\xi|^{2} t} L \widehat{E_{0 \perp}},
\end{gathered}
$$


where $M$ and $L$ denote the following matrices (in the three dimensional case),

$$
M=\frac{1}{|\xi|^{2}}\left(\begin{array}{ccc}
\xi_{1}^{2} & \xi_{1} \xi_{2} & \xi_{1} \xi_{3} \\
\xi_{1} \xi_{2} & \xi_{2}^{2} & \xi_{2} \xi_{3} \\
\xi_{1} \xi_{3} & \xi_{2} \xi_{3} & \xi_{3}^{2}
\end{array}\right) L=\frac{1}{|\xi|^{2}}\left(\begin{array}{ccc}
\xi_{2}^{2}+\xi_{3}^{2} & -\xi_{1} \xi_{2} & -\xi_{1} \xi_{3} \\
-\xi_{1} \xi_{2} & \xi_{1}^{2}+\xi_{3}^{2} & -\xi_{2} \xi_{3} \\
-\xi_{1} \xi_{3} & -\xi_{2} \xi_{3} & \xi_{1}^{2}+\xi_{2}^{2}
\end{array}\right)=I-M
$$

Remark 1. For $E \in L^{2}\left(\mathbb{R}^{N}\right)$ satisfying $\nabla \cdot E=0$ then $M \widehat{E}=0$ and $L \widehat{E}=\widehat{E}$. That is

$$
\mathcal{F}^{-1}(M \widehat{E})=\mathcal{P}_{\|} E \mathcal{F}^{-1}(L \widehat{E})=\mathcal{P}_{\perp} E .
$$

So, we have

$$
\begin{aligned}
S_{\|}(t) E_{0 \|} & =\mathcal{F}^{-1}(M) * \mathcal{F}^{-1}\left(e^{-i|\xi|^{2} t}\right) *\left(E_{0 \|}\right)=\mathcal{F}^{-1}\left(e^{-i|\xi|^{2} t}\right) *\left(E_{0 \|}\right), \\
S_{\perp}(t) E_{0 \perp} & =\mathcal{F}^{-1}(L) * \mathcal{F}^{-1}\left(e^{-i \alpha^{2}|\xi|^{2} t}\right) *\left(E_{0 \perp}\right)=\mathcal{F}^{-1}\left(e^{-i \alpha^{2}|\xi|^{2} t}\right) *\left(E_{0 \perp}\right) .
\end{aligned}
$$

We then remark that the group $S_{\|}(t)$ is associated to

$$
\begin{gathered}
i E_{\|_{t}}+\triangle E_{\|}=0 \\
E_{\| \mid t=0}=E_{0 \|} \in L^{2}\left(\mathbb{R}^{N}\right),
\end{gathered}
$$

and $S_{\perp}(t)$ is associated to

$$
\begin{gathered}
i E_{\perp t}+\alpha^{2} \triangle E_{\perp}=0 \\
E_{\perp \mid t=0}=E_{0 \perp} \in L^{2}\left(\mathbb{R}^{N}\right) .
\end{gathered}
$$

We return to the expression of $S_{\|}(t)$ and $S_{\perp}(t)$ in the Fourier space. It is well known that

$$
\mathcal{F}^{-1}\left(e^{-i \alpha^{2}|\xi|^{2} t}\right)=\frac{1}{\left(4 \pi i \alpha^{2} t\right)^{\frac{N}{2}}} e^{i \frac{|x|^{2}}{4 \alpha^{2} t}}
$$

It then follows

$$
\begin{aligned}
S_{\|}(t) E_{0 \|} & =\frac{1}{(4 \pi i t)^{\frac{N}{2}}} \int_{\mathbb{R}^{N}} e^{i \frac{|x-y|^{2}}{4 t}} E_{0 \|}(y) \mathrm{d} y, \\
S_{\perp}(t) E_{0 \perp} & =\frac{1}{\left(4 \pi i \alpha^{2} t\right)^{\frac{N}{2}}} \int_{\mathbb{R}^{N}} e^{i \frac{|x-y|^{2}}{4 \alpha^{2} t}} E_{0 \perp}(y) \mathrm{d} y .
\end{aligned}
$$

From these last expressions and as the $S_{\|}(t)$ and $S_{\perp}(t)$ are unitary groups on $L^{2}\left(\mathbb{R}^{N}\right)$, we have

$$
\left|S_{\|}(t) E_{0 \|}\right|_{L^{2}\left(\mathbb{R}^{N}\right)}=\left|E_{0 \|}\right|_{L^{2}\left(\mathbb{R}^{N}\right)},\left|S_{\|}(t) E_{0 \|}\right|_{L^{\infty}\left(\mathbb{R}^{N}\right)} \leq \frac{C}{(4 \pi t)^{\frac{N}{2}}}\left|E_{0 \|}\right|_{L^{1}\left(\mathbb{R}^{N}\right)}
$$

and

$$
\left|S_{\perp}(t) E_{0 \perp}\right|_{L^{2}\left(\mathbb{R}^{N}\right)}=\left|E_{0 \perp}\right|_{L^{2}\left(\mathbb{R}^{N}\right)},\left|S_{\perp}(t) E_{0 \perp}\right|_{L^{\infty}\left(\mathbb{R}^{N}\right)} \leq \frac{C}{\left(4 \pi \alpha^{2} t\right)^{\frac{N}{2}}}\left|E_{0 \perp}\right|_{L^{1}\left(\mathbb{R}^{N}\right)} .
$$

By interpolation, we get for $p>2$,

$$
\left|S_{\|}(t) E_{0 \|}\right|_{L^{p}\left(\mathbb{R}^{N}\right)} \leq \frac{C}{(4 \pi t)^{N\left(\frac{1}{2}-\frac{1}{p}\right)}}\left|E_{0 \|}\right|_{L^{p^{\prime}}\left(\mathbb{R}^{N}\right)}
$$


and

$$
\left|S_{\perp}(t) E_{0 \perp}\right|_{L^{p}\left(\mathbb{R}^{N}\right)} \leq \frac{C}{\left(4 \pi \alpha^{2} t\right)^{N\left(\frac{1}{2}-\frac{1}{p}\right)}}\left|E_{0 \perp}\right|_{L^{p^{\prime}}\left(\mathbb{R}^{N}\right)}
$$

with $\frac{1}{p}+\frac{1}{p^{\prime}}=1$. This last estimate is fundamental since the right-hand side goes to zero when $\alpha$ goes to infinity $(p>2)$.

\subsection{Estimates with a right-hand side}

Using the same splitting as the one used for (1.5), the solution of

$$
\begin{gathered}
i E_{t}+\nabla \nabla \cdot E+\alpha^{2}(\triangle-\nabla \nabla .) E=f, \\
E_{\mid t=0}=E_{0}
\end{gathered}
$$

where $f$ depends on $t \in \mathbb{R}$ and $x \in \mathbb{R}^{3}$, can be splitted in two equations,

$$
\begin{gathered}
i E_{\|_{t}}+\triangle E_{\|}=\varepsilon \mathcal{P}_{\|} f \text { in } \mathcal{D}^{\prime}\left(\mathbb{R}^{N}\right), \\
E_{\|_{\mid t=0}}=\mathcal{P}_{\|} E_{0} \in L^{2}\left(\mathbb{R}^{N}\right), \\
i E_{\perp t}+\alpha^{2} \triangle E_{\perp}=\varepsilon \mathcal{P}_{\perp} f \text { in } \mathcal{D}^{\prime}\left(\mathbb{R}^{N}\right), \\
E_{\perp \mid t=0}=\mathcal{P}_{\perp} E_{0} \in L^{2}\left(\mathbb{R}^{N}\right) .
\end{gathered}
$$

We then have,

$$
\begin{aligned}
& E_{\|}(t)=S_{\|}(t) E_{0 \|}-i \int_{0}^{t} S_{\|}(t-s) \mathcal{P}_{\|}(f(s)) \mathrm{d} s \\
& E_{\perp}(t)=S_{\perp}(t) E_{0 \perp}-i \int_{0}^{t} S_{\perp}(t-s) \mathcal{P}_{\perp}(f(s)) \mathrm{d} s .
\end{aligned}
$$

The following result will be often used in the sequel:

Lemma 2.1. (see for example [6], p. 109). The operators $\mathcal{P}_{\|}, \mathcal{P}_{\perp}$ are continuous on $L^{p}\left(\mathbb{R}^{N}\right)$ for all $1<p<\infty$.

We also introduce the

Lemma 2.2. (see [6], p. 119). We denote

$$
I_{a}(f)=\int_{\mathbb{R}^{d}} \frac{1}{|x-y|^{d-a}} f(y) \mathrm{d} y,
$$

for $0<a<d$ and $1<p<q<\infty$, where $\frac{1}{q}=\frac{1}{p}-\frac{a}{d}$. Then, there exists a constant $A_{p, q}$ such that,

$$
\left|I_{a}(f)\right|_{L^{q}\left(\mathbb{R}^{d}\right)} \leq A_{p, q}|f|_{L^{p}\left(\mathbb{R}^{d}\right)}
$$

Proposition 2.1. Let $(q, r)$ two real numbers such that,

$$
\frac{1}{2} \leq r<\frac{2 N}{N-2}, \frac{2}{q}=\frac{N}{2}-\frac{n}{r}
$$

and let $\left(q^{\prime}, r^{\prime}\right)$ be the conjugate exponents of $(q, r)$. 
If $f \in L^{q^{\prime}}\left(\left[0, T\left[; L^{r^{\prime}}\left(\mathbb{R}^{N}\right)\right)\right.\right.$ then

$$
\int_{0}^{t} S_{\|}(t-s) \mathcal{P}_{\|}(f(s)) \mathrm{d} s \in \mathcal{C}\left([0, T], L^{2}\left(\mathbb{R}^{N}\right)\right) \cap L^{q}\left(\left[0, T\left[; L^{r}\left(\mathbb{R}^{N}\right)\right)\right.\right.
$$

and

$$
\int_{0}^{t} S_{\perp}(t-s) \mathcal{P}_{\perp}(f(s)) \mathrm{d} s \in \mathcal{C}\left([0, T], L^{2}\left(\mathbb{R}^{N}\right)\right) \cap L^{q}\left(\left[0, T\left[; L^{r}\left(\mathbb{R}^{N}\right)\right) .\right.\right.
$$

Furthermore, there exists a real constant $C$ depending only on $q$ and $r$ such that,

$$
\begin{gathered}
\left|\int_{0}^{t} S_{\|}(t-s) \mathcal{P}_{\|}(f(s)) \mathrm{d} s\right|_{\mathcal{C}\left([0, T] ; L^{2}\left(\mathbb{R}^{N}\right)\right) \cap L^{q}\left(\left[0, T\left[; L^{r}\left(\mathbb{R}^{N}\right)\right)\right.\right.} \leq C|f|_{L^{q^{\prime}}\left(\left[0, T\left[; L^{\left.r^{\prime}\left(\mathbb{R}^{N}\right)\right)}\right.\right.\right.}, \\
\left|\int_{0}^{t} S_{\perp}(t-s) \mathcal{P}_{\perp}(f(s)) \mathrm{d} s\right|_{\mathcal{C}\left([0, T] ; L^{2}\left(\mathbb{R}^{N}\right)\right) \cap L^{q}\left(\left[0, T\left[; L^{r}\left(\mathbb{R}^{N}\right)\right)\right.\right.} \leq C \alpha^{-N\left(1-\frac{2}{r}\right)}|f|_{L^{q^{\prime}}\left(\left[0, T\left[; L^{r^{\prime}}\left(\mathbb{R}^{N}\right)\right)\right.\right.} .
\end{gathered}
$$

At last, if $E_{0} \in L^{2}\left(\mathbb{R}^{N}\right)$, then

$$
\left|S_{\|}(t) \mathcal{P}_{\|}\left(E_{0}\right)\right|_{L^{q}\left(\left[0, T\left[; L^{r}\left(\mathbb{R}^{N}\right)\right)\right.\right.} \leq C\left|\mathcal{P}_{\|}\left(E_{0}\right)\right|_{L^{2}\left(\mathbb{R}^{N}\right)}
$$

and

$$
\left|S_{\perp}(t) E_{0 \perp}\right|_{L^{q}\left(\left[0, T\left[; L^{r}\left(\mathbb{R}^{N}\right)\right)\right.\right.} \leq C \alpha^{-N\left(1-\frac{2}{r}\right)}\left|E_{0 \perp}\right|_{L^{2}\left(\mathbb{R}^{N}\right)} .
$$

Proof. According to (2.5), one has

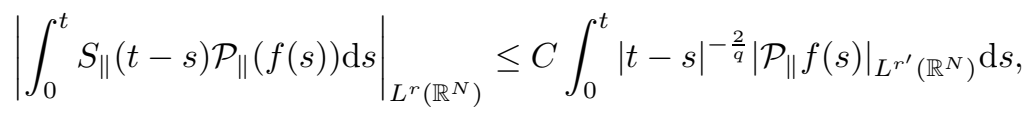

since $\frac{2}{q}=\frac{N}{2}-\frac{N}{r}$.

In the same way, from (2.6), we have

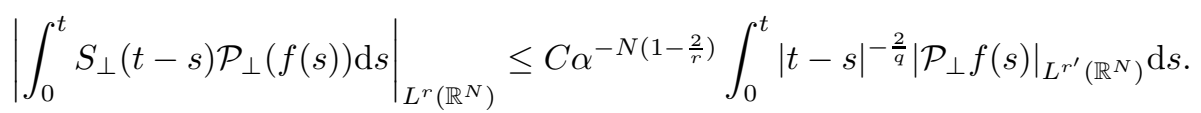

It follows from Lemma 2.2 the first estimate of the proposition 2.1, (with $d=1, \frac{2}{q}=1-a$ then $\frac{1}{q}=\frac{1}{q^{\prime}}-a$, so $\left.p=q^{\prime}\right)$,

We now compute

$$
\left|\int_{0}^{t} S_{\|}(t-s) \mathcal{P}_{\|}(f(s)) \mathrm{d} s\right|_{L^{q}\left(\left[0, T\left[; L^{r}\left(\mathbb{R}^{N}\right)\right)\right.\right.} \leq C\left|\mathcal{P}_{\|} f\right|_{L^{q^{\prime}}\left(\left[0, T\left[; L^{r^{\prime}}\left(\mathbb{R}^{N}\right)\right)\right.\right.}
$$

$$
\begin{aligned}
\left|\int_{0}^{t} S_{\|}(t-s) \mathcal{P}_{\|}(f(s)) \mathrm{d} s\right|_{L^{2}\left(\mathbb{R}^{N}\right)}^{2} & =\int_{0}^{t} \int_{0}^{t}\left\langle S_{\|}(t-s) \mathcal{P}_{\|}(f(s)), S_{\|}\left(t-s^{\prime}\right) \mathcal{P}_{\|}\left(f\left(s^{\prime}\right)\right)\right\rangle \mathrm{d} s \mathrm{~d} s^{\prime} \\
& =\int_{0}^{t}\left\langle\mathcal{P}_{\|}(f(s)), \int_{0}^{t} S_{\|}\left(s-s^{\prime}\right) \mathcal{P}_{\|}\left(f\left(s^{\prime}\right)\right) \mathrm{d} s^{\prime}\right\rangle \mathrm{d} s
\end{aligned}
$$

where the brackets $\langle.,$.$\rangle denotes the classical inner product on L^{2}\left(\mathbb{R}^{N}\right)$. We can show as previously that

$$
\left.\int_{0}^{t} S_{\|}\left(s-s^{\prime}\right) \mathcal{P}_{\|}\left(f\left(s^{\prime}\right)\right) \mathrm{d} s^{\prime}\right|_{L^{q}\left(\left[0, T\left[; L^{r}\left(\mathbb{R}^{N}\right)\right)\right.\right.} \leq C\left|\mathcal{P}_{\|} f\right|_{L^{q^{\prime}}\left(\left[0, T\left[; L^{r^{\prime}}\left(\mathbb{R}^{N}\right)\right)\right.\right.},
$$


then,

$$
\left|\int_{0}^{t} S_{\|}(t-s) \mathcal{P}_{\|}(f(s)) \mathrm{d} s\right|_{L^{2}\left(\mathbb{R}^{N}\right)}^{2} \leq C|f|_{L^{q^{\prime}}\left(\left[0, T\left[; L^{r^{\prime}}\left(\mathbb{R}^{N}\right)\right)\right.\right.}^{2}
$$

To show the estimate

$$
\left.\left|S_{\|}(t) E_{0 \|}\right|_{L^{q}\left(\left[0, T\left[; L^{r}\left(\mathbb{R}^{N}\right)\right)\right.\right.} \leq C \mid E_{0 \|}\right)\left.\right|_{L^{2}\left(\mathbb{R}^{N}\right)},
$$

we proceed by duality,

$$
\begin{aligned}
\left|\int_{0}^{T} S_{\|}(-t) \mathcal{P}_{\|}(f(t)) \mathrm{d} t\right|_{L^{2}\left(\mathbb{R}^{N}\right)}^{2} & =\int_{0}^{T} \int_{0}^{T}\left\langle\mathcal{P}_{\|} f(t), S_{\|}\left(t-t^{\prime}\right) \mathcal{P}_{\|}\left(f\left(t^{\prime}\right)\right)\right\rangle \mathrm{d} t \mathrm{~d} t^{\prime} \\
& \leq C|f|_{L^{q^{\prime}}\left(\left[0, T\left[; L^{r^{\prime}}\left(\mathbb{R}^{N}\right)\right)\right.\right.}^{2} .
\end{aligned}
$$

Then,

$$
\begin{aligned}
\left|\int_{0}^{T}\left\langle S_{\|}(t)\left(\mathcal{P}_{\|} E_{0}\right), \mathcal{P}_{\|}(f(t))\right\rangle \mathrm{d} t\right| & =\left|\left\langle E_{0 \|}, \int_{0}^{T} S_{\|}(-t) \mathcal{P}_{\|}(f(t)) \mathrm{d} t\right\rangle\right| \\
& \leq\left|\mathcal{P}_{\|} E_{0}\right|_{L^{2}\left(\mathbb{R}^{N}\right)}\left|\int_{0}^{T} S_{\|}(-t) \mathcal{P}_{\|}(f(t)) \mathrm{d} t\right|_{L^{2}\left(\mathbb{R}^{N}\right)}^{2} \\
& \leq C\left|\mathcal{P}_{\|} E_{0}\right|_{L^{2}\left(\mathbb{R}^{N}\right)}\left|\mathcal{P}_{\|} f\right|_{L^{q^{\prime}}\left(\left[0, T\left[; L^{r^{\prime}}\left(\mathbb{R}^{N}\right)\right)\right.\right.} .
\end{aligned}
$$

We then have proved,

$$
\left|S_{\|}(t)\left(E_{0 \|}\right)\right|_{L^{q}\left(\left[0, T\left[; L^{r}\left(\mathbb{R}^{N}\right)\right)\right.\right.} \leq C\left|E_{0 \|}\right|_{L^{2}\left(\mathbb{R}^{N}\right)} .
$$

Due to the similarities between $(2.11)$ and (2.10), the same estimates for the group $S_{\perp}(t)$ can be obtained by replacing $C$ with $C \alpha^{-N\left(1-\frac{2}{r}\right)}$. In the same way, $\mathcal{P}_{\|}$is replaced with $\mathcal{P}_{\perp}$.

We then have proved the estimates involving $S_{\perp}(t)$ of Proposition 2.1.

\section{The Cauchy problem}

Let us recall the problem under studies,

$$
\begin{gathered}
i E_{t}+\nabla \nabla \cdot E+\alpha^{2}(\triangle-\nabla \nabla .) E=\varepsilon|E|^{2 \sigma} E \text { in } \mathcal{D}^{\prime}\left(\mathbb{R}^{N}\right), \\
E_{\mid t=0}=E_{0} \in L^{2}\left(\mathbb{R}^{N}\right) .
\end{gathered}
$$

This system is splitted as follows,

$$
\begin{aligned}
i E_{\|_{t}}+\triangle E_{\|}= & \varepsilon \mathcal{P}_{\|}\left(\left|E_{\|}+E_{\perp}\right|^{2 \sigma}\left(E_{\|}+E_{\perp}\right)\right) \text { in } \mathcal{D}^{\prime}\left(\mathbb{R}^{N}\right) \\
& E_{\|_{\mid t=0}}=\mathcal{P}_{\|} E_{0} \in L^{2}\left(\mathbb{R}^{N}\right)
\end{aligned}
$$

and

$$
\begin{gathered}
i E_{\perp t}+\alpha^{2} \triangle E_{\perp}=\varepsilon \mathcal{P}_{\perp}\left(\left|E_{\|}+E_{\perp}\right|^{2 \sigma}\left(E_{\|}+E_{\perp}\right)\right) \text { in } \mathcal{D}^{\prime}\left(\mathbb{R}^{N}\right) \\
E_{\perp \mid t=0}=\mathcal{P}_{\perp} E_{0} \in L^{2}\left(\mathbb{R}^{N}\right) .
\end{gathered}
$$




\subsection{Global existence in $L^{2}\left(\mathbb{R}^{N}\right)$}

Theorem 3.1. Assume that $\sigma<\frac{2}{N}$ and $E_{0} \in L^{2}\left(\mathbb{R}^{N}\right)$; then there exists a unique solution $E$ of (3.1) in $\mathcal{C}\left(\mathbb{R}^{+} ; L^{2}\left(\mathbb{R}^{N}\right)\right) \cap L_{\text {loc }}^{q}\left(\mathbb{R}^{+} ; L^{2 \sigma+2}\left(\mathbb{R}^{N}\right)\right)$, where $\frac{2}{q}=\frac{N}{2}-\frac{N}{2 \sigma+2}$.

Also, $E$ depends continuously on $E_{0}$ in the sense: if $E_{0}^{n} \rightarrow E_{0}$ in $L^{2}\left(\mathbb{R}^{N}\right)$ then $E^{n}$, the solution of (3.1) with the initial data $E_{0}^{n}$ converge to $E$ in

$$
\mathcal{C}_{\text {loc }}\left(\mathbb{R}^{+} ; L^{2}\left(\mathbb{R}^{N}\right)\right) \cap L_{\text {loc }}^{q}\left(\mathbb{R}^{+} ; L^{2 \sigma+2}\left(\mathbb{R}^{N}\right)\right) .
$$

At last, E verifies

$$
\left|\mathcal{P}_{\perp} E\right|_{L^{q}\left(\left[0, T\left[; L^{2 \sigma+2}\left(\mathbb{R}^{N}\right)\right)\right.\right.} \leq C \alpha^{-\frac{N \sigma}{\sigma+1}}\left|E_{0 \perp}\right|_{L^{2}\left(\mathbb{R}^{N}\right)}+C(T) \alpha^{-\frac{N \sigma}{\sigma+1}}|E|_{L^{q}\left(\left[0, T\left[; L^{2 \sigma+2}\left(\mathbb{R}^{N}\right)\right)\right.\right.}^{2 \sigma+1} .
$$

Proof. We first establish the local existence of a solution by a fixed point theorem.

Let us introduce the space

$$
X(T)=L^{\infty}\left(\left[0, T\left[; L^{2}\left(\mathbb{R}^{N}\right)\right) \cap L^{q}\left(\left[0, T\left[; L^{2 \sigma+2}\left(\mathbb{R}^{N}\right)\right) .\right.\right.\right.\right.
$$

We note $\mathcal{T}$ the application

$$
\mathcal{T}: \begin{aligned}
& X(T) \times X(T) \rightarrow X(T) \times X(T) \\
& \left(E_{\|}, E_{\perp}\right) \rightarrow\left(\mathcal{T}_{1}\left(E_{\|}, E_{\perp}\right), \mathcal{T}_{2}\left(E_{\|}, E_{\perp}\right)\right)
\end{aligned}
$$

where

$$
\begin{aligned}
& \mathcal{T}_{1}\left(E_{\|}, E_{\perp}\right)=S_{\|}(T) E_{0 \|}-i \varepsilon \int_{0}^{T} S_{\|}(T-s)\left(\mathcal{P}_{\|}\left(\left|E_{\|}+E_{\perp}\right|^{2 \sigma}\left(E_{\|}+E_{\perp}\right)\right)\right) \mathrm{d} s, \\
& \mathcal{T}_{2}\left(E_{\|}, E_{\perp}\right)=S_{\perp}(T) E_{0 \perp}-i \varepsilon \int_{0}^{T} S_{\perp}(T-s)\left(\mathcal{P}_{\perp}\left(\left|E_{\|}+E_{\perp}\right|^{2 \sigma}\left(E_{\|}+E_{\perp}\right)\right)\right) \mathrm{d} s .
\end{aligned}
$$

It follows from Proposition 2.1 that

$$
\left|\mathcal{T}_{1}\left(E_{\|}, E_{\perp}\right)\right|_{X(T)} \leq C\left|E_{0 \|}\right|_{L^{2}\left(\mathbb{R}^{N}\right)}+C \| E_{\|}+\left.\left.E_{\perp}\right|^{2 \sigma}\left(E_{\|}+E_{\perp}\right)\right|_{L^{q^{\prime}}\left(\left[0, T\left[; L^{\left.(2 \sigma+2)^{\prime}\left(\mathbb{R}^{N}\right)\right)}\right.\right.\right.}
$$

and

$$
\begin{aligned}
\left|\mathcal{T}_{2}\left(E_{\|}, E_{\perp}\right)\right|_{X(T)} \leq & \left(1+C \alpha^{-\frac{N \sigma}{\sigma+1}}\right)\left|E_{0 \perp}\right|_{L^{2}\left(\mathbb{R}^{N}\right)} \\
& +C \alpha^{-\frac{N \sigma}{\sigma+1}}|| E_{\|}+\left.\left.E_{\perp}\right|^{2 \sigma}\left(E_{\|}+E_{\perp}\right)\right|_{L^{q^{\prime}}\left(\left[0, T\left[; L^{(2 \sigma+2)^{\prime}}\left(\mathbb{R}^{N}\right)\right)\right.\right.} .
\end{aligned}
$$

Adding these two last inequalities for $\alpha \geq 1$ for example, we have,

$$
\begin{aligned}
& \left|\mathcal{T}_{1}\left(E_{\|}, E_{\perp}\right)\right|_{X(T)}+\left|\mathcal{T}_{2}\left(E_{\|}, E_{\perp}\right)\right|_{X(T)} \leq \\
& \quad C\left(\left|E_{0 \|}\right|_{L^{2}\left(\mathbb{R}^{N}\right)}+\left|E_{0 \perp}\right|_{L^{2}\left(\mathbb{R}^{N}\right)}+|| E_{\|}+\left.\left.E_{\perp}\right|^{2 \sigma}\left(E_{\|}+E_{\perp}\right)\right|_{L^{q^{\prime}}\left(\left[0, T\left[; L^{\left.(2 \sigma+2)^{\prime}\left(\mathbb{R}^{N}\right)\right)}\right) .\right.\right.}\right.
\end{aligned}
$$

Remark that $(2 \sigma+2)^{\prime}=(2 \sigma+2) \frac{1}{2 \sigma+1}$, then,

$$
|| E_{\|}+\left.\left.E_{\perp}\right|^{2 \sigma}\left(E_{\|}+E_{\perp}\right)\right|_{L^{q^{\prime}}\left(\left[0, T\left[; L^{\left.(2 \sigma+2)^{\prime}\left(\mathbb{R}^{N}\right)\right)}\right.\right.\right.}=\left|E_{\|}+E_{\perp}\right|_{L^{q^{\prime}(2 \sigma+1)}\left(\left[0, T\left[; L^{2 \sigma+2}\left(\mathbb{R}^{N}\right)\right)\right.\right.}^{2 \sigma+1} .
$$

As $\sigma<\frac{2}{N}$, after straightforward computations, we can verify that

$$
q^{\prime}(2 \sigma+1)<q .
$$


Then, there exists $\theta>0$ such that, by virtue of Hölder inequality

$$
\left|E_{\|}+E_{\perp}\right|_{L^{q^{\prime}(2 \sigma+1)}\left(\left[0, T\left[; L^{2 \sigma+2}\left(\mathbb{R}^{N}\right)\right)\right.\right.} \leq T^{\theta}\left|E_{\|}+E_{\perp}\right|_{L^{q}\left(\left[0, T\left[; L^{2 \sigma+2}\left(\mathbb{R}^{N}\right)\right)\right.\right.} \leq T^{\theta}\left|E_{\|}+E_{\perp}\right|_{X(T)} .
$$

Returning to (3.6), we obtain

$$
\left|\mathcal{T}_{1}\left(E_{\|}, E_{\perp}\right)\right|_{X(T)}+\left|\mathcal{T}_{2}\left(E_{\|}, E_{\perp}\right)\right|_{X(T)} \leq C\left(\left|E_{0 \|}\right|_{L^{2}\left(\mathbb{R}^{N}\right)}+\left|E_{0 \perp}\right|_{L^{2}\left(\mathbb{R}^{N}\right)}+T^{(2 \sigma+1) \theta}\left(\left|E_{\|}\right|_{X(T)}^{2 \sigma+1}+\left|E_{\perp}\right|_{X(T)}^{2 \sigma+1}\right)\right.
$$

Lemma 3.1. If $T$ is small enough, then $\mathcal{T}$ operates from the ball $B(0, R)$ of $X(T) \times X(T)$ into itself as soon as

$$
R \leq 4 C\left|E_{0}\right|_{L^{2}\left(\mathbb{R}^{N}\right)}
$$

Proof. With $R$ introduced in Lemma 3.1, inequality (3.7) becomes

$$
\left|\mathcal{T}\left(E_{\|}, E_{\perp}\right)\right|_{X(T) \times X(T)} \leq \frac{R}{2}+2 C T^{(2 \sigma+1) \theta}\left|\left(E_{\|}, E_{\perp}\right)\right|_{X(T) \times X(T)} .
$$

So, for $T$ small enough, the Lemma 3.1 is proved.

Lemma 3.2. If $T$ is small enough, then $\mathcal{T}$ is a contraction on $B_{R}$.

Proof. Let $\left(E_{\|_{1}}, E_{\perp_{1}}\right)$ and $\left(E_{\|_{2}}, E_{\perp_{2}}\right)$ belonging to $X(T) \times X(T)$,

$$
\begin{aligned}
\left|\mathcal{T}_{1}\left(E_{\|_{1}}, E_{\perp 1}\right)-\mathcal{T}_{1}\left(E_{\|_{2}}, E_{\perp 2}\right)\right|_{X(T)}+\left|\mathcal{T}_{2}\left(E_{\|_{1}}, E_{\perp 1}\right)-\mathcal{T}_{2}\left(E_{\|_{2}}, E_{\perp 2}\right)\right|_{X(T)} \\
\quad \leq C|| E_{\|_{1}}+\left.E_{\perp 1}\right|^{2 \sigma}\left(E_{\|_{1}}+E_{\perp 1}\right)-\left.\left|E_{\|_{2}}+E_{\perp 2}\right|^{2 \sigma}\left(E_{\|_{2}}+E_{\perp_{2}}\right)\right|_{L^{q^{\prime}}\left(\left[0, T\left[; L^{(2 \sigma+2)^{\prime}}\left(\mathbb{R}^{N}\right)\right)\right.\right.}
\end{aligned}
$$

As,

we have,

$$
\left.|| u\right|^{2 \sigma} u-|v|^{2 \sigma} v\left|\leq(2 \sigma+1)\left(|u|^{2 \sigma}+|v|^{2 \sigma}\right)\right| u-v \mid
$$

$$
\begin{aligned}
\left|\mathcal{T}_{1}\left(E_{\|_{1}}, E_{\perp 1}\right)-\mathcal{T}_{1}\left(E_{\|_{2}}, E_{\perp 2}\right)\right|_{X(T)}+\left|\mathcal{T}_{2}\left(E_{\|_{1}}, E_{\perp 1}\right)-\mathcal{T}_{2}\left(E_{\|_{2}}, E_{\perp 2}\right)\right|_{X(T)} \\
\quad \leq C\left|\left(\left|E_{1}\right|^{2 \sigma}+\left|E_{2}\right|^{2 \sigma}\right)\left(\left|E_{\|_{1}}-E_{\|_{2}}\right|+\left|E_{\perp 1}-E_{\perp 2}\right|\right)\right|_{L^{q^{\prime}}\left(\left[0, T\left[; L^{(2 \sigma+2)^{\prime}}\left(\mathbb{R}^{N}\right)\right)\right.\right.} .
\end{aligned}
$$

From Hölder inequality, we obtain,

$$
\begin{aligned}
\left|\mathcal{T}_{1}\left(E_{\|_{1}}, E_{\perp 1}\right)-\mathcal{T}_{1}\left(E_{\|_{2}}, E_{\perp 2}\right)\right|_{X(T)}+\left|\mathcal{T}_{2}\left(E_{\|_{1}}, E_{\perp 1}\right)-\mathcal{T}_{2}\left(E_{\|_{2}}, E_{\perp 2}\right)\right|_{X(T)} \\
\quad \leq\left. C|| E_{1}\right|^{2 \sigma}+\left.\left|E_{2}\right|^{2 \sigma}\right|_{L^{q_{1}}\left(\left[0, T\left[; L^{\gamma}\right)\right.\right.}\left(\left|E_{\|_{1}}-E_{\|_{2}}\right|_{L^{q_{2}}\left(\left[0, T\left[; L^{\beta}\right)\right.\right.}+\left|E_{\perp 1}-E_{\perp 2}\right|_{L^{q_{2}}\left(\left[0, T\left[L^{\beta}\right)\right.\right.}\right)
\end{aligned}
$$

with $\frac{1}{\gamma}+\frac{1}{\beta}=\frac{1}{(2 \sigma+2)^{\prime}}, \frac{1}{q_{1}}+\frac{1}{q_{2}}=\frac{1}{q^{\prime}}$.

We choose $\gamma=\frac{2 \sigma+2}{2 \sigma}$ then $\beta=2 \sigma+2$ and we take $q_{1}=\frac{q}{2 \sigma}$.

Then, as $\sigma<\frac{2}{N}$, we can verify that

Then, there exists $\theta>0$ such that

$$
q_{2}=\frac{q^{\prime} q}{q-2 \sigma q^{\prime}}<q
$$

$$
\begin{aligned}
\left|\mathcal{T}_{1}\left(E_{\|_{1}}, E_{\perp_{1}}\right)-\mathcal{T}_{1}\left(E_{\|_{2}}, E_{\perp_{2}}\right)\right|_{X(T)}+\left|\mathcal{T}_{2}\left(E_{\|_{1}}, E_{\perp_{1}}\right)-\mathcal{T}_{2}\left(E_{\|_{2}}, E_{\perp_{2}}\right)\right|_{X(T)} \\
\quad \leq C T^{\theta}\left(\left|E_{\|_{1}}+E_{\perp_{1}}\right|_{X(T)}^{2 \sigma}+\left|E_{\|_{2}}+E_{\perp_{2}}\right|_{X(T)}^{2 \sigma}\right)\left(\left|E_{\|_{1}}-E_{\|_{2}}\right|_{X(T)}+\left|E_{\perp_{1}}-E_{\perp_{2}}\right|_{X(T)}\right)
\end{aligned}
$$

This last estimate with $T$ small enough ensures the Lemma 3.2. 
The existence of a solution in $X(T)$ follows from the Banach fixed point theorem. Furthermore $T$ depends only on $\left|E_{0}\right|_{L^{2}\left(\mathbb{R}^{N}\right)}(\sigma$ fixed).

In fact $T=+\infty$ because the $L^{2}\left(\mathbb{R}^{N}\right)$-norm of $E(t)=E_{\|}(t)+E_{\perp}(t)$ is constant for all $t$. This is obtained, formally, by inner product of (3.1) with $\bar{E}_{t}$.

At this step, we know that $\mathcal{T}_{2}\left(E_{\|}, E_{\perp}\right)=E_{\perp}$, then the estimates (3.5) shows (3.4). This ends the proof of Theorem 3.1.

\subsection{Local or global existence in $H^{1}\left(\mathbb{R}^{N}\right)$}

This section is devoted to the existence of solution for $(3.1)$ in $H^{1}\left(\mathbb{R}^{N}\right)$.

Theorem 3.2. Assume that $\sigma<\frac{2}{N-2}$ and $E_{0} \in H^{1}\left(\mathbb{R}^{N}\right)$; then there exist $T_{\alpha}>0$ and a unique maximal solution of (3.1) in $\mathcal{C}\left(\left[0, T_{\alpha}\left[; H^{1}\left(\mathbb{R}^{N}\right)\right) \cap L_{\mathrm{loc}}^{q}\left(\left[0, T_{\alpha}\left[; W^{1,2 \sigma+2}\left(\mathbb{R}^{N}\right)\right)\right.\right.\right.\right.$, where $\frac{2}{q}=\frac{N}{2}-\frac{N}{2 \sigma+2}$. Furthermore, if $T_{\alpha}<\infty$, then

$$
\lim _{t \rightarrow T_{\alpha}^{-}}|E(t)|_{H^{1}\left(\mathbb{R}^{N}\right)}=+\infty .
$$

Also, $E$ depends continuously on $E_{0}$ in the sense: if $E_{0}^{n} \rightarrow E_{0}$ in $H^{1}\left(\mathbb{R}^{N}\right)$ then $E^{n}$, the solution of (3.1) with the initial data $E_{0}^{n}$ converge to $E$ in

$$
\mathcal{C}\left(\left[0, T\left[; H^{1}\left(\mathbb{R}^{N}\right)\right) \cap L^{q}\left(\left[0, T\left[; W^{1,2 \sigma+2}\left(\mathbb{R}^{N}\right)\right) \forall T<T_{\alpha} .\right.\right.\right.\right.
$$

At last the following estimate holds

$$
\left|\nabla \mathcal{P}_{\perp} E\right|_{L^{q}\left(\left[0, T\left[; L^{2 \sigma+2}\left(\mathbb{R}^{N}\right)\right)\right.\right.} \leq C \alpha^{-\frac{N \sigma}{\sigma+1}}\left|\nabla E_{0 \perp}\right|_{L^{2}\left(\mathbb{R}^{N}\right)}+C(T) \alpha^{-\frac{N \sigma}{\sigma+1}}|E|_{Y(T))}^{2 \sigma+1},
$$

with

$$
Y(T)=\{E \text { such that } E \in X(T), \nabla E \in X(T)\} .
$$

Proof. We keep the same notations as in the proof of Theorem 3.1. As before, we assume that $\alpha \geq 1$. We prove this theorem by a fixed point method on the space $Y(T) \times Y(T)$. We saw that

$$
\begin{aligned}
& \left|\mathcal{T}_{1}\left(E_{\|}, E_{\perp}\right)\right|_{X(T)}+\left|\mathcal{T}_{2}\left(E_{\|}, E_{\perp}\right)\right|_{X(T)} \leq
\end{aligned}
$$

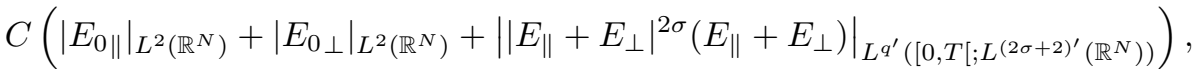

and we remark that, since $(2 \sigma+2)^{\prime-1}=\frac{2 \sigma}{2 \sigma+2}+\frac{1}{2 \sigma+2}$.

$$
\left.\left.|| E\right|^{2 \sigma}(E)\right|_{L^{q^{\prime}}\left(\left[0, T\left[; L^{\left.(2 \sigma+2)^{\prime}\left(\mathbb{R}^{N}\right)\right)}\right.\right.\right.} \leq\left.\left.|| E\right|_{L^{2 \sigma+2}\left(\mathbb{R}^{N}\right)} ^{2 \sigma}\right|_{L^{\infty}(0, T)}|E|_{L^{q^{\prime}}\left(\left[0, T\left[; L^{2 \sigma+2}\left(\mathbb{R}^{N}\right)\right)\right.\right.} .
$$

As $H^{1}\left(\mathbb{R}^{N}\right)$ imbeds in $L^{2 \sigma+2}\left(\mathbb{R}^{N}\right)$, as soon as $\sigma<\frac{2}{N-2}$, we have

$$
\begin{aligned}
\left|\mathcal{T}_{1}\left(E_{\|}, E_{\perp}\right)\right|_{X(T)}+\left|\mathcal{T}_{2}\left(E_{\|}, E_{\perp}\right)\right|_{X(T)} & \leq \\
& C\left(\left|E_{0 \|}\right|_{L^{2}\left(\mathbb{R}^{N}\right)}+\left|E_{0 \perp}\right|_{L^{2}\left(\mathbb{R}^{N}\right)}+\left|E_{\|}+E_{\perp}\right|_{L^{\infty}\left(\left[0, T\left[; H^{1}\left(\mathbb{R}^{N}\right)\right)\right.\right.}^{2 \sigma}\left|E_{\|}+E_{\perp}\right|_{L^{q^{\prime}}\left(\left[0, T\left[; L^{2 \sigma+2}\left(\mathbb{R}^{N}\right)\right)\right.\right.}\right)
\end{aligned}
$$

Then, from Hölder's inequality, we obtain,

$$
\begin{aligned}
\left|\mathcal{T}_{1}\left(E_{\|}, E_{\perp}\right)\right|_{X(T)}+\mid & \left.\mathcal{T}_{2}\left(E_{\|}, E_{\perp}\right)\right|_{X(T)} \\
& \leq\left(\left|E_{0 \|}\right|_{L^{2}\left(\mathbb{R}^{N}\right)}+\left|E_{0 \perp}\right|_{L^{2}\left(\mathbb{R}^{N}\right)}+\left|E_{\|}+E_{\perp}\right|_{L^{\infty}\left(\left[0, T\left[; H^{1}\left(\mathbb{R}^{N}\right)\right)\right.\right.}^{2 \sigma} T^{\frac{1}{q^{\prime}}-\frac{1}{q}}\left|E_{\|}+E_{\perp}\right|_{X(T)}\right) .
\end{aligned}
$$


As,

$$
\mid \nabla\left(\left|E_{\|}+E_{\perp}\right|^{2 \sigma}\left(E_{\|}+E_{\perp}\right)|\leq(2 \sigma+1)| E_{\|}+\left.E_{\perp}\right|^{2 \sigma}\left|\nabla\left(E_{\|}+E_{\perp}\right)\right|\right.
$$

an estimate on $\nabla \mathcal{T}_{1}\left(E_{\|}, E_{\perp}\right)$ and $\nabla \mathcal{T}_{2}\left(E_{\|}, E_{\perp}\right)$ with $X(T)$-norm leads, in the same way as $(3.11)$, to

$$
\begin{aligned}
& \left|\nabla \mathcal{T}_{1}\left(E_{\|}, E_{\perp}\right)\right|_{X(T)}+\left|\nabla \mathcal{T}_{2}\left(E_{\|}, E_{\perp}\right)\right|_{X(T)} \\
& \quad \leq C\left(\left|\nabla E_{0 \|}\right|_{L^{2}\left(\mathbb{R}^{N}\right)}+\left|\nabla E_{0 \perp}\right|_{L^{2}\left(\mathbb{R}^{N}\right)}+|E|_{L^{\infty}\left(\left[0, T\left[; H^{1}\left(\mathbb{R}^{N}\right)\right)\right.\right.}^{2 \sigma} T^{\frac{1}{q^{\prime}}-\frac{1}{q}}|\nabla E|_{X(T)}\right)
\end{aligned}
$$

Estimates (3.11) and (3.12) establish the

Lemma 3.3. Let $R^{\prime}=4 C\left(\left|E_{0 \|}\right|_{H^{1}\left(\mathbb{R}^{N}\right)}+\left|E_{0 \perp}\right|_{H^{1}\left(\mathbb{R}^{N}\right)}\right)$, if $T$ is small enough, the application $\mathcal{T}$ operates from the ball $B\left(0, R^{\prime}\right)$ of $(Y(T) \times Y(T))$ into itself.

Lemma 3.4. If $T$ is small enough, $\mathcal{T}$ is a contraction on $B_{R^{\prime}}(Y(T) \times Y(T))$.

Proof. Let $\left(E_{\|_{1}}, E_{\perp 1}\right)$ and $\left(E_{\|_{2}}, E_{\perp 2}\right)$ belonging to $Y(T) \times Y(T)$. Starting from (3.8) and using the same idea as for the proof of Lemma 3.3, we have

$$
\begin{aligned}
\left|\mathcal{T}_{1}\left(E_{\|_{1}}, E_{\perp 1}\right)-\mathcal{T}_{1}\left(E_{\|_{2}}, E_{\perp 2}\right)\right|_{X(T)}+\left|\mathcal{T}_{2}\left(E_{\|_{1}}, E_{\perp 1}\right)-\mathcal{T}_{2}\left(E_{\|_{2}}, E_{\perp 2}\right)\right|_{X(T)} & \\
& \leq C\left(\sum_{i=1}^{2}\left|E_{\|_{i}}+E_{\perp i}\right|_{L^{\infty}\left(\left[0, T\left[; H^{1}\left(\mathbb{R}^{N}\right)\right)\right.\right.}^{2 \sigma}\right) T^{\frac{1}{q^{\prime}}-\frac{1}{q}}\left(\left|E_{\|_{1}}-E_{\|_{2}}\right|_{X(T)}+\left|E_{\perp 1}-E_{\perp 2}\right|_{X(T)}\right)
\end{aligned}
$$

At this step, we have shown that, for $T$ small enough, $\mathcal{T}$ is a contraction on $B_{R^{\prime}}(Y(T) \times Y(T))$ for the $X(T) \times X(T)$ topology. The Theorem 3.2 is thus proved by fixed point method on $X(T) \times X(T)$. In fact, we can estimate $\mathcal{T}_{1}\left(E_{\|_{1}}, E_{\perp_{1}}\right)-\mathcal{T}_{1}\left(E_{\|_{2}}, E_{\perp_{2}}\right)$ with the $Y(T) \times Y(T)$-norm,

$$
\begin{gathered}
\left|\nabla\left(\mathcal{T}_{1}\left(E_{\|_{1}}, E_{\perp 1}\right)-\mathcal{T}_{1}\left(E_{\|_{2}}, E_{\perp 2}\right)\right)\right|_{X(T)}+\left|\nabla\left(\mathcal{T}_{2}\left(E_{\|_{1}}, E_{\perp 1}\right)-\mathcal{T}_{2}\left(E_{\|_{2}}, E_{\perp 2}\right)\right)\right|_{X(T)} \\
\leq C\left|\nabla f\left(E_{\|_{1}}+E_{\perp 1}\right)-\nabla f\left(E_{\|_{2}}+E_{\perp 2}\right)\right|_{L^{q^{\prime}}\left(\left[0, T\left[; L^{(2 \sigma+2)^{\prime}}\left(\mathbb{R}^{N}\right)\right)\right.\right.}
\end{gathered}
$$

with $f(u)=|u|^{2 \sigma} u$.

Remark that

$$
\begin{aligned}
\left|f^{\prime}(u)\right| & \leq(2 \sigma+1)|u|^{2 \sigma} \\
\left|f^{\prime \prime}(u)\right| & \leq 2 \sigma(2 \sigma+1)|u|^{2 \sigma-1}
\end{aligned}
$$

Let us write $u=E_{\|_{1}}+E_{\perp 1}$ and $v=E_{\|_{2}}+E_{\perp 2}$,

$$
\begin{aligned}
\nabla f(u)-\nabla f(v) & =f^{\prime}(u) \nabla u+f^{\prime}(v) \nabla v=f^{\prime}(u)(\nabla u-\nabla v)\left(f^{\prime}(u)-f^{\prime}(v)\right) \nabla v \\
& =f^{\prime}(u)(\nabla u-\nabla v)+\int_{0}^{1} f^{\prime \prime}(u+\theta(v-u)(v-u) \mathrm{d} \theta \nabla v
\end{aligned}
$$

So, since $\frac{2 \sigma}{2 \sigma+2}+\frac{1}{2 \sigma+2}=\frac{1}{(2 \sigma+2)^{\prime}}$ and $\frac{2 \sigma-1}{2 \sigma+2}+\frac{1}{2 \sigma+2}+\frac{1}{2 \sigma+2}=\frac{1}{(2 \sigma+2)^{\prime}}$,

$$
\begin{aligned}
& |\nabla f(u)-\nabla f(v)|_{L^{q^{\prime}}\left(\left[0, T\left[; L^{(2 \sigma+2)^{\prime}}\right)\right.\right.} \leq C\left|f^{\prime}(u)\right|_{L^{\infty}\left(\left[0, T\left[; L^{\frac{2 \sigma+2}{2 \sigma}}\right)\right.\right.}|\nabla f(u)-\nabla f(v)|_{L^{q^{\prime}}\left(\left[0, T\left[; L^{2 \sigma+2}\right)\right.\right.} \\
& \quad+C \sup _{\theta \in(0,1)}\left|f^{\prime \prime}(u+\theta(v-u))\right|_{L^{\infty}\left(\left[0, T\left[; L^{\frac{2 \sigma+2}{2 \sigma-1}}\right)\right.\right.}|v-u|_{L^{\infty}\left(\left[0, T\left[; L^{2 \sigma+2}\right)\right.\right.}|\nabla v|_{L^{q^{\prime}}\left(\left[0, T\left[; L^{2 \sigma+2}\right)\right.\right.}
\end{aligned}
$$


Using this result in (3.13), we obtain, by virtue of (3.14),

$$
\begin{aligned}
\mid \nabla \mathcal{T}_{1}\left(E_{\|_{1}}, E_{\perp 1}\right) & -\left.\nabla \mathcal{T}_{1}\left(E_{\|_{2}}, E_{\perp 2}\right)\right|_{X(T)}+\left|\nabla \mathcal{T}_{2}\left(E_{\|_{1}}, E_{\perp 1}\right)-\nabla \mathcal{T}_{2}\left(E_{\|_{2}}, E_{\perp 2}\right)\right|_{X(T)} \\
& \leq C\left|E_{\|_{1}}+E_{\perp 1}\right|_{L^{\infty}\left(\left[0, T\left[; L^{2 \sigma+2}\left(\mathbb{R}^{N}\right)\right)\right.\right.}^{2 \sigma}\left|\nabla\left(E_{\|_{1}}-E_{\|_{2}}\right)+\nabla\left(E_{\perp 1}-E_{\perp 2}\right)\right|_{L^{q^{\prime}}\left(\left[0, T\left[; L^{2 \sigma+2}\left(\mathbb{R}^{N}\right)\right)\right.\right.} \\
& +C \sum_{i=1}^{2}\left|E_{\|_{i}}+E_{\perp_{i}}\right|_{L^{\infty}\left(\left[0, T\left[; L^{2 \sigma+2}\left(\mathbb{R}^{N}\right)\right)\right.\right.}\left|\nabla\left(E_{\|_{2}}+E_{\perp 2}\right)\right|_{L^{q^{\prime}}\left(\left[0, T\left[; L^{2 \sigma+2}\left(\mathbb{R}^{N}\right)\right)\right.\right.} \\
& \times\left(\left|E_{\|_{1}}-E_{\|_{2}}\right|_{L^{\infty}\left(\left[0, T\left[; L^{2 \sigma+2}\left(\mathbb{R}^{N}\right)\right)\right.\right.}+\left|E_{\perp 1}-E_{\perp_{2}}\right|_{L^{\infty}\left(\left[0, T\left[; L^{2 \sigma+2}\left(\mathbb{R}^{N}\right)\right)\right.\right.}\right) .
\end{aligned}
$$

As $H^{1}\left(\mathbb{R}^{N}\right)$ imbeds in $L^{2 \sigma+2}\left(\mathbb{R}^{N}\right)$, and from Hölder inequality, we obtain

$$
\begin{aligned}
\mid \nabla \mathcal{T}_{1}\left(E_{\|_{1}}, E_{\perp 1}\right) & -\left.\nabla \mathcal{T}_{1}\left(E_{\|_{2}}, E_{\perp_{2}}\right)\right|_{X(T)}+\left|\nabla \mathcal{T}_{2}\left(E_{\|_{1}}, E_{\perp_{1}}\right)-\nabla \mathcal{T}_{2}\left(E_{\|_{2}}, E_{\perp_{2}}\right)\right|_{X(T)} \\
& \leq C\left(\left|E_{\|_{1}}+E_{\perp_{1}}\right|_{L^{\infty}\left(\left[0, T\left[; H^{1}\right)\right.\right.}^{2 \sigma}+\sum_{i=1}^{2}\left|E_{\|_{i}}+E_{\perp_{i}}\right|_{L^{\infty}\left(\left[0, T\left[; H^{1}\right)\right.\right.}^{2 \sigma-1}\left|\nabla\left(E_{\|_{2}}+E_{\perp_{2}}\right)\right|_{L^{q}\left(\left[0, T\left[; L^{2 \sigma+2}\right)\right.\right.}\right) \\
& \times T^{\frac{1}{q^{\prime}}-\frac{1}{q}}\left(\left|E_{\|_{1}}-E_{\|_{2}}\right|_{Y(T)}+\left|E_{\perp 1}-E_{\perp 2}\right|_{Y(T)}\right) .
\end{aligned}
$$

This ensures that, for $T$ small enough, $\mathcal{T}$ is a contraction on $B_{R^{\prime}}(Y(T) \times Y(T))$ for the $Y(T) \times Y(T)$ topology. Then, the Banach fixed point theorem shows that $E$ depends continuously on the initial data $E_{0}$ for the $Y(T)$ topology.

The estimate (3.9) follows from Proposition 2.1, as the estimate (3.12). The Theorem 3.2 is then proved.

\section{Theorem 3.3.}

- If $\varepsilon=1$ and $\sigma<\frac{2}{N-2}$ then the solution of (3.1) with $E_{0} \in H^{1}\left(\mathbb{R}^{N}\right)$ belongs to $\mathcal{C}\left(\left[0, \infty\left[; H^{1}\left(\mathbb{R}^{N}\right)\right) \cap\right.\right.$ $L_{\text {loc }}^{q}\left(\left[0, \infty\left[; W^{1,2 \sigma+2}\left(\mathbb{R}^{N}\right)\right)\right.\right.$, where $\frac{2}{q}=\frac{N}{2}-\frac{N}{2 \sigma+2}$.

- If $\varepsilon=-1$ and $\sigma<\frac{2}{N}$, then the solution of (3.1) with $E_{0} \in H^{1}\left(\mathbb{R}^{N}\right)$ belongs to $\mathcal{C}\left(\left[0, \infty\left[; H^{1}\left(\mathbb{R}^{N}\right)\right) \cap\right.\right.$ $L_{\text {loc }}^{q}\left(\left[0, \infty\left[; W^{1,2 \sigma+2}\left(\mathbb{R}^{N}\right)\right)\right.\right.$, where $\frac{2}{q}=\frac{N}{2}-\frac{N}{2 \sigma+2}$.

Proof. These results follows from the energy conservation,

$$
\begin{aligned}
\left|\nabla E_{\|}(t)\right|_{L^{2}\left(\mathbb{R}^{N}\right)}^{2}+\alpha^{2}\left|\nabla E_{\perp}(t)\right|_{L^{2}\left(\mathbb{R}^{N}\right)}^{2}+ & \frac{\varepsilon}{2} \frac{1}{2 \sigma+2}\left|E_{\|}(t)+E_{\perp}(t)\right|_{L^{2 \sigma+2}\left(\mathbb{R}^{N}\right)}^{2 \sigma+2}= \\
& \left|\nabla E_{0 \|}\right|_{L^{2}\left(\mathbb{R}^{N}\right)}^{2}+\alpha^{2}\left|\nabla E_{0 \perp}\right|_{L^{2}\left(\mathbb{R}^{N}\right)}^{2}+\frac{\varepsilon}{2} \frac{1}{2 \sigma+2}\left|E_{0 \|}+E_{0 \perp}\right|_{L^{2 \sigma+2}\left(\mathbb{R}^{N}\right)}^{2 \sigma+2}
\end{aligned}
$$

This last is obtained, formally, by taking the real part of the inner product of (3.2) with $\overline{E_{\|_{t}}}$,

$$
-\frac{\mathrm{d}}{\mathrm{d} t}\left|\nabla E_{\|}\right|_{L^{2}\left(\mathbb{R}^{N}\right)}^{2}=\varepsilon \int_{\mathbb{R}^{N}} \mathcal{P}_{\|}\left(|E|^{2 \sigma} E\right) \overline{E_{\|_{t}}}=\varepsilon \int_{\mathbb{R}^{N}}|E|^{2 \sigma} E \overline{E_{\|_{t}}},
$$

by taking the real part of the inner product of (3.3) with $\overline{E_{\perp t}}$,

$$
-\alpha^{2} \frac{\mathrm{d}}{\mathrm{d} t}\left|\nabla E_{\perp}\right|_{L^{2}\left(\mathbb{R}^{N}\right)}^{2}=\varepsilon \int_{\mathbb{R}^{N}} \mathcal{P}_{\perp}\left(|E|^{2 \sigma} E\right) \overline{E_{\perp t}}=\varepsilon \int_{\mathbb{R}^{N}}|E|^{2 \sigma} E \overline{E_{\perp t}} .
$$

Then, adding and integrating these two estimates, we obtain $(3.15)$. $\bullet$ The first part of the theorem $(\varepsilon=1)$ is then obvious, since the solution, bounded in $H^{1}\left(\mathbb{R}^{N}\right) \subset L^{2 \sigma+2}\left(\mathbb{R}^{N}\right)$, can not blow up.

- Assume that $\varepsilon=-1$, we have

$$
\left|\nabla E_{\|}(t)\right|_{L^{2}\left(\mathbb{R}^{N}\right)}^{2}+\alpha^{2}\left|\nabla E_{\perp}(t)\right|_{L^{2}\left(\mathbb{R}^{N}\right)}^{2}=C+\frac{1}{4 \sigma+4}\left|E_{\|}(t)+E_{\perp}(t)\right|_{L^{2 \sigma+2}\left(\mathbb{R}^{N}\right)}^{2 \sigma+2} .
$$


We recall the Gagliardo-Nirenberg inequality,

Lemma 3.5. Let $u$ be in $H^{1}\left(\mathbb{R}^{N}\right)$,

$$
|u|_{L^{2 \sigma+2}\left(\mathbb{R}^{N}\right)}^{2 \sigma+2} \leq c|\nabla u|_{L^{2}\left(\mathbb{R}^{N}\right)}^{N \sigma}|u|_{L^{2}\left(\mathbb{R}^{N}\right)}^{2((2-N) \sigma+2)} .
$$

Assume that $\sigma<\frac{2}{N}$, then, since the $L^{2}\left(\mathbb{R}^{N}\right)$-norm of $E$ is conserved,

$$
\left|E_{\|}(t)+E_{\perp}(t)\right|_{L^{2 \sigma+2}\left(\mathbb{R}^{N}\right)}^{2 \sigma+2} \leq c\left|\nabla\left(E_{\|}(t)+E_{\perp}(t)\right)\right|_{L^{2}\left(\mathbb{R}^{N}\right)}^{\mu}\left|E_{0}\right|_{L^{2}\left(\mathbb{R}^{N}\right)}^{2((2-N) \sigma+2)},
$$

with $\mu<2$. Finally, if $\alpha \geq 1$, the estimate (3.16) leads to

$$
|\nabla E|_{L^{2}\left(\mathbb{R}^{N}\right)}^{2} \leq\left|\nabla E_{\|}(t)\right|_{L^{2}\left(\mathbb{R}^{N}\right)}^{2}+\alpha^{2}\left|\nabla E_{\perp}(t)\right|_{L^{2}\left(\mathbb{R}^{N}\right)}^{2} \leq c^{\prime}|\nabla E|_{L^{2}\left(\mathbb{R}^{N}\right)}^{\mu}+C(\alpha)
$$

Then, assume that $E$ blows up in $H^{1}\left(\mathbb{R}^{N}\right)$, this is clearly impossible by virtue of the following inequality,

$$
|\nabla E|_{L^{2}\left(\mathbb{R}^{N}\right)}^{\frac{2}{\mu}} \leq c^{\prime}+\frac{C(\alpha)}{|\nabla E|_{L^{2}\left(\mathbb{R}^{N}\right)}^{\mu}} .
$$

Then, the second part of Theorem 3.3 is proved.

\section{THE CONVERGENCE WHEN $\alpha$ GOES TO INFINITY}

The estimates (3.4) and (3.9) are the main point to study the convergence of the solution when $\alpha$ goes to infinity.

\subsection{Convergence in $L^{2}\left(\mathbb{R}^{N}\right)$}

Theorem 4.1. Assume $\sigma<\frac{2}{N}$. Let us denote $E_{\alpha}$ the solution of (3.1), passing to the limit, we have

$$
\begin{gathered}
E_{\alpha} \rightarrow E \text { in } L^{q}\left(\left[0, T\left[; L^{2 \sigma+2}\left(\mathbb{R}^{N}\right)\right) \text { strong }\left(\frac{2}{q}=\frac{N}{2}-\frac{N}{2 \sigma+2}\right),\right.\right. \\
E_{\alpha}-S_{\perp}(.)\left(E_{0 \perp}\right) \rightarrow E \text { in } L^{\infty}\left(\left[0, T\left[; L^{2}\left(\mathbb{R}^{N}\right)\right) \forall T<+\infty\right.\right.
\end{gathered}
$$

where E verifies

$$
\begin{aligned}
i E_{t}+\triangle E & =\mathcal{P}_{\|}\left(|E|^{2 \sigma} E\right) \\
E_{\mid t=0} & =E_{0 \|} .
\end{aligned}
$$

Proof. Remark that $E$, the solution of (4.1) can be estimated as for the solution of (3.1), that is to say, $E$ belongs to $L^{q}\left(\left[0, T\left[; L^{2 \sigma+2}\left(\mathbb{R}^{N}\right)\right) \cap L^{\infty}\left(\left[0, T\left[; L^{2}\left(\mathbb{R}^{N}\right)\right)\right.\right.\right.\right.$ for all $T>0$.

Let us modify the notations of the previous sections. The solutions of (3.1), (3.2) and (3.3) are indexed by $\alpha$ $\left(E_{\alpha}, E_{\alpha \|}\right.$ and $E_{\alpha \perp}$ respectively):

$$
\begin{gathered}
i E_{\alpha t}+\nabla \nabla \cdot E_{\alpha}+\alpha^{2}(\triangle-\nabla \nabla .) E_{\alpha}=\varepsilon\left|E_{\alpha}\right|^{2 \sigma} E_{\alpha} \text { in } \mathcal{D}^{\prime}\left(\mathbb{R}^{N}\right) \\
E_{\alpha \mid t=0}=E_{0} \in L^{2}\left(\mathbb{R}^{N}\right) .
\end{gathered}
$$


This system is splitted as follows,

$$
\begin{aligned}
i E_{\alpha \|_{t}}+\triangle E_{\alpha \|} & =\varepsilon \mathcal{P}_{\|}\left(\left|E_{\alpha \|}+E_{\alpha \perp}\right|^{2 \sigma}\left(E_{\alpha \|}+E_{\alpha \perp}\right)\right) \text { in } \mathcal{D}^{\prime}\left(\mathbb{R}^{N}\right), \\
E_{\alpha \|_{\mid t=0}} & =\mathcal{P}_{\|} E_{0} \in L^{2}\left(\mathbb{R}^{N}\right), \\
i E_{\alpha \perp t}+\alpha^{2} \triangle E_{\alpha \perp} & =\varepsilon \mathcal{P}_{\perp}\left(\left|E_{\alpha \|}+E_{\alpha \perp}\right|^{2 \sigma}\left(E_{\alpha \|}+E_{\alpha \perp}\right)\right) \text { in } \mathcal{D}^{\prime}\left(\mathbb{R}^{N}\right), \\
E_{\alpha \perp \mid t=0} & =\mathcal{P}_{\perp} E_{0} \in L^{2}\left(\mathbb{R}^{N}\right) .
\end{aligned}
$$

In the previous section, with (3.4), we have shown that

$$
E_{\alpha \perp} \rightarrow 0 \text { in } L^{q}\left(\left[0, T\left[; L^{2 \sigma+2}\left(\mathbb{R}^{N}\right)\right) .\right.\right.
$$

Also,

$$
E_{\alpha \perp}-S_{\perp}(.) E_{0 \perp} \rightarrow 0 \text { in } L^{\infty}\left(\left[0, T\left[; L^{2}\left(\mathbb{R}^{N}\right)\right) .\right.\right.
$$

To establish the theorem, we just have to prove that

$$
E_{\alpha \|} \rightarrow E \text { in } L^{q}\left(\left[0, T\left[; L^{2 \sigma+2}\left(\mathbb{R}^{N}\right)\right) \cap L^{\infty}\left(\left[0, T\left[; L^{2}\left(\mathbb{R}^{N}\right)\right) .\right.\right.\right.\right.
$$

Let $D_{\alpha}=E_{\alpha \|}-E, D_{\alpha}$ verifies the partial differential system

$$
\begin{gathered}
i D_{\alpha t}+\triangle D_{\alpha}=\mathcal{P}_{\|}\left(\left|E_{\alpha \|}+E_{\alpha \perp}\right|^{2 \sigma}\left(E_{\alpha \|}+E_{\alpha \perp}\right)-|E|^{2 \sigma} E\right) \\
D_{\alpha \mid t=0}=0 .
\end{gathered}
$$

This last is equivalent to

$$
D_{\alpha}(t)=\int_{0}^{t} S_{\|}(t-s) \mathcal{P}_{\|}\left(\left|E_{\alpha \|}+E_{\alpha \perp}\right|^{2 \sigma}\left(E_{\alpha \|}+E_{\alpha \perp}\right)-|E|^{2 \sigma} E\right)(s) \mathrm{d} s .
$$

Applying the proposition 2.1, we have

$$
\left|D_{\alpha}\right|_{L^{q}\left(\left[0, T\left[; L^{2 \sigma+2}\right) \cap L^{\infty}\left(\left[0, T\left[; L^{2}\left(\mathbb{R}^{N}\right)\right)\right.\right.\right.\right.} \leq C|| E_{\alpha \|}+\left.E_{\alpha \perp}\right|^{2 \sigma}\left(E_{\alpha \|}+E_{\alpha \perp}\right)-\left.|E|^{2 \sigma} E\right|_{L^{q^{\prime}}\left(\left[0, T\left[; L^{(2 \sigma+2)^{\prime}}\right)\right.\right.} .
$$

The right-hand side is estimated in the same way as in the previous section to establish the Lemma 3.2 .

$$
\begin{aligned}
& || E_{\alpha \|}+\left.E_{\alpha \perp}\right|^{2 \sigma}\left(E_{\alpha \|}+E_{\alpha \perp}\right)-\left.|E|^{2 \sigma} E\right|_{L^{q^{\prime}}\left(\left[0, T\left[; L^{\left.(2 \sigma+2)^{\prime}\left(\mathbb{R}^{N}\right)\right)}\right.\right.\right.} \leq \\
& c|| E_{\alpha \|}+\left.E_{\alpha \perp}\right|^{2 \sigma}+\left.|E|^{2 \sigma}\right|_{L^{q_{1}\left(\left[0, T\left[; L^{\gamma}\right)\right.\right.}}\left|D_{\alpha}+E_{\alpha \perp}\right|_{L^{q_{2}}\left(\left[0, T\left[; L^{\beta}\right)\right.\right.},
\end{aligned}
$$

with $\frac{1}{\gamma}+\frac{1}{\beta}=\frac{1}{(2 \sigma+2)^{\prime}}, \frac{1}{q_{1}}+\frac{1}{q_{2}}=\frac{1}{q^{\prime}}$.

We choose $\gamma=\frac{2 \sigma+2}{2 \sigma}$ then $\beta=2 \sigma+2$ and we take $q_{1}=\frac{q}{2 \sigma}$.

Then, as $\sigma<\frac{2}{N}$ we can verify that

Then, there exists $\theta=\frac{1}{q_{2}}-\frac{1}{q}>0$ such that

$$
q_{2}=\frac{q^{\prime} q}{q-2 \sigma q^{\prime}}<q
$$

$$
\begin{aligned}
& \left|D_{\alpha}\right|_{L^{q}\left(\left[0, T\left[; L^{2 \sigma+2}\right) \cap L^{\infty}\left(\left[0, T\left[; L^{2}\right)\right.\right.\right.\right.} \leq \\
& \qquad T^{\theta}\left(\left|E_{\alpha}\right|_{L^{q}\left(\left[0, T\left[; L^{2 \sigma+2}\right)\right.\right.}^{2 \sigma}+|E|_{L^{q}\left(\left[0, T\left[; L^{2 \sigma+2}\right)\right.\right.}^{2 \sigma}\right)\left(\left|D_{\alpha}\right|_{L^{q}\left(\left[0, T\left[; L^{2 \sigma+2}\right)\right.\right.}+\left|E_{\alpha \perp}\right|_{L^{q}\left(\left[0, T\left[; L^{2 \sigma+2}\right)\right.\right.}\right) .
\end{aligned}
$$


That is, if $T \leq T_{m}<\infty$,

$$
\left|D_{\alpha}\right|_{L^{q}\left(\left[0, T\left[; L^{2 \sigma+2}\left(\mathbb{R}^{N}\right)\right) \cap L^{\infty}\left(\left[0, T\left[; L^{2}\left(\mathbb{R}^{N}\right)\right)\right.\right.\right.\right.} \leq C\left(T_{m}\right) T^{\theta}\left(\left|D_{\alpha}\right|_{L^{q}\left(\left[0, T\left[; L^{2 \sigma+2}\left(\mathbb{R}^{N}\right)\right)\right.\right.}+\left|E_{\alpha \perp}\right|_{L^{q}\left(\left[0, T\left[; L^{2 \sigma+2}\left(\mathbb{R}^{N}\right)\right)\right.\right.}\right) .
$$

So, if we choose $T$ small enough,

$$
\left|D_{\alpha}\right|_{L^{q}\left(\left[0, T\left[; L^{2 \sigma+2}\left(\mathbb{R}^{N}\right)\right) \cap L^{\infty}\left(\left[0, T\left[; L^{2}\left(\mathbb{R}^{N}\right)\right)\right.\right.\right.\right.} \leq \frac{1}{2}\left|D_{\alpha}\right|_{L^{q}\left(\left[0, T\left[; L^{2 \sigma+2}\left(\mathbb{R}^{N}\right)\right)\right.\right.}+\frac{1}{2}\left|E_{\alpha \perp}\right|_{L^{q}\left(\left[0, T\left[; L^{2 \sigma+2}\left(\mathbb{R}^{N}\right)\right)\right.\right.} .
$$

That is,

$$
\left|D_{\alpha}\right|_{L^{q}\left(\left[0, T\left[; L^{2 \sigma+2}\left(\mathbb{R}^{N}\right)\right) \cap L^{\infty}\left(\left[0, T\left[; L^{2}\left(\mathbb{R}^{N}\right)\right)\right.\right.\right.\right.} \leq\left|E_{\alpha \perp}\right|_{L^{q}\left(\left[0, T\left[; L^{2 \sigma+2}\left(\mathbb{R}^{N}\right)\right)\right.\right.} .
$$

We then have shown that $D_{\alpha}$ goes to zero when $\alpha$ goes to infinity on $L^{q}\left(\left[0, T\left[; L^{2 \sigma+2}\left(\mathbb{R}^{N}\right)\right) \cap L^{\infty}\left(\left[0, T\left[; L^{2}\left(\mathbb{R}^{N}\right)\right)\right.\right.\right.\right.$ if $T$ is small enough. In fact, we have the same results for all $T<\infty$. Assume that there exists $T_{m}<\infty$ such that

$$
\begin{aligned}
& D_{\alpha} \rightarrow 0 \text { in } L^{q}\left(\left[0, T\left[; L^{2 \sigma+2}\left(\mathbb{R}^{N}\right)\right) \cap L^{\infty}\left(\left[0, T\left[; L^{2}\left(\mathbb{R}^{N}\right)\right) \forall T<T_{m}\right.\right.\right.\right. \\
& D_{\alpha} \nrightarrow 0 \text { in } L^{q}\left(\left[0, T_{m}+\eta\left[; L^{2 \sigma+2}\left(\mathbb{R}^{N}\right)\right) \cap L^{\infty}\left(\left[0, T_{m}+\eta\left[; L^{2}\left(\mathbb{R}^{N}\right)\right) \forall \eta>0 .\right.\right.\right.\right.
\end{aligned}
$$

As previously, we show that there exits $\delta>0$ such that

$$
\left|D_{\alpha}\right|_{L^{q}\left(\left[T, T+\delta\left[; L^{2 \sigma+2}\right) \cap L^{\infty}\left(\left[T, T+\delta\left[; L^{2}\right)\right.\right.\right.\right.} \leq C\left|D_{\alpha}(T)\right|_{L^{2}}+\left|E_{\alpha \perp}\right|_{L^{q}\left(\left[T, T+\delta\left[; L^{2 \sigma+2}\right)\right.\right.} .
$$

From the first part of (4.5), we know that:

$$
\begin{aligned}
D_{\alpha}(T) & \rightarrow 0 \text { in } L^{2}\left(\mathbb{R}^{N}\right),\left(T<T_{m}\right) \\
E_{\alpha \perp} & \rightarrow 0 \text { in } L^{q}\left(\left[T, T+\delta\left[; L^{2 \sigma+2}\left(\mathbb{R}^{N}\right)\right) .\right.\right.
\end{aligned}
$$

Then, let us choose $T=T_{m}-\frac{\delta}{2}$ and the second relation of (4.5) is false. The Theorem 4.1 is so proved.

\subsection{Convergence in $H^{1}\left(\mathbb{R}^{N}\right)$}

Let us now formulate a result on convergence of solution of (4.2) when $\alpha$ goes to infinity in $H^{1}\left(\mathbb{R}^{N}\right)$.

Theorem 4.2. Assume that $\sigma<\frac{2}{N-2}$, if $\mathcal{P}_{\perp} E_{0} \rightarrow 0$ in $H^{1}\left(\mathbb{R}^{N}\right)$ when $\alpha$ goes to infinity, then

$$
\begin{aligned}
& E_{\alpha} \rightarrow E \text { in } W^{1, q}\left(\left[0, T\left[; L^{2 \sigma+2}\left(\mathbb{R}^{N}\right)\right) \text { strong }\left(\frac{2}{q}=\frac{N}{2}-\frac{N}{2 \sigma+2}\right),\right.\right. \\
& E_{\alpha} \rightarrow E \text { in } L^{\infty}\left(\left[0, T\left[; H^{1}\left(\mathbb{R}^{N}\right)\right) \forall T<T^{*}\right.\right.
\end{aligned}
$$

where E verifies (4.1) and $T^{*}$ is the existence time of the solution of (4.1) in

$$
\mathcal{C}\left(\left[0, T\left[; H^{1}\left(\mathbb{R}^{N}\right)\right) \cap W^{1, q}\left(\left[0, T\left[; L^{2 \sigma+2}\left(\mathbb{R}^{N}\right)\right),\right.\right.\right.\right.
$$

see theorem 1.1, also $\liminf _{\alpha \rightarrow+\infty} T_{\alpha} \geq T^{*}$.

If $\varepsilon=1$ or if $\varepsilon=-1$ and $\sigma<\frac{2}{N}$ then $T^{*}=+\infty$.

Proof. Remark that $E$, the solution of $(4.1)$ is well defined in $L^{\infty}\left(\left[0, T\left[; H^{1}\left(\mathbb{R}^{N}\right)\right) \cap W^{1, q}\left(\left[0, T\left[; L^{2 \sigma+2}\left(\mathbb{R}^{N}\right)\right)\right.\right.\right.\right.$, for $T$ (eventually) small enough. This can be shown as for $E_{\alpha \|}$, see [2].

In the previous section, with (3.9), we have shown that

$$
E_{\alpha \perp} \rightarrow 0 \text { in } W^{1, q}\left(\left[0, T\left[; L^{2 \sigma+2}\left(\mathbb{R}^{N}\right)\right) \forall T<T^{*}\right.\right.
$$


Also,

$$
E_{\alpha \perp}-S_{\perp}(t) E_{0 \perp} \rightarrow 0 \text { in } L^{\infty}\left(\left[0, T\left[; H^{1}\left(\mathbb{R}^{N}\right)\right) \forall T<T^{*} .\right.\right.
$$

It remains to be shown that

$$
E_{\alpha \|} \rightarrow E \text { in } W^{1, q}\left(\left[0, T\left[; L^{2 \sigma+2}\left(\mathbb{R}^{N}\right)\right) \cap L^{\infty}\left(\left[0, T\left[; H^{1}\left(\mathbb{R}^{N}\right)\right) \forall T<T^{*} .\right.\right.\right.\right.
$$

We introduce again $D_{\alpha}=E_{\alpha \|}-E$,

$$
D_{\alpha}(t)=\int_{0}^{t} S_{\|}(t-s) \mathcal{P}_{\|}\left(\left|E_{\alpha \|}+E_{\alpha \perp}\right|^{2 \sigma}\left(E_{\alpha \|}+E_{\alpha \perp}\right)-|E|^{2 \sigma} E\right)(s) \mathrm{d} s .
$$

The proposition 2.1 ensures that

$$
\left|D_{\alpha}\right|_{L^{q}\left(\left[0, T\left[; L^{2 \sigma+2}\left(\mathbb{R}^{N}\right)\right) \cap L^{\infty}\left(\left[0, T\left[; L^{2}\left(\mathbb{R}^{N}\right)\right)\right.\right.\right.\right.} \leq\left. C|| E_{\alpha}\right|^{2 \sigma}\left(E_{\alpha}\right)-\left.|E|^{2 \sigma} E\right|_{L^{q^{\prime}}\left(\left[0, T\left[; L^{(2 \sigma+2)^{\prime}}\left(\mathbb{R}^{N}\right)\right)\right.\right.} .
$$

That is,

$$
\begin{aligned}
& \left|D_{\alpha}\right|_{L^{q}\left(\left[0, T\left[; L^{2 \sigma+2}\left(\mathbb{R}^{N}\right)\right) \cap L^{\infty}\left(\left[0, T\left[; L^{2}\left(\mathbb{R}^{N}\right)\right)\right.\right.\right.\right.} \leq\left. C\left|\left(\left|E_{\alpha}\right|^{2 \sigma}+|E|^{2 \sigma}\right)\right| D_{\alpha}\right|_{L^{q^{\prime}}\left(\left[0, T\left[; L^{(2 \sigma+2)^{\prime}}\left(\mathbb{R}^{N}\right)\right)\right.\right.} \\
& \quad \leq C\left(\left|E_{\alpha}\right|_{L^{\infty}\left(\left[0, T\left[; L^{2 \sigma+2}\left(\mathbb{R}^{N}\right)\right)\right.\right.}^{2 \sigma}+|E|_{L^{\infty}\left(\left[0, T\left[; L^{2 \sigma+2}\left(\mathbb{R}^{N}\right)\right)\right.\right.}^{2 \sigma}\right)\left|E_{\alpha}-E\right|_{L^{q^{\prime}}\left(\left[0, T\left[; L^{2 \sigma+2}\left(\mathbb{R}^{N}\right)\right)\right.\right.} .
\end{aligned}
$$

As $H^{1}\left(\mathbb{R}^{N}\right) \subset L^{2 \sigma+2}\left(\mathbb{R}^{N}\right)\left(\sigma<\frac{2}{N-2}\right)$, and from Hölder inequality, we have

$$
\begin{aligned}
& \left|D_{\alpha}\right|_{L^{q}\left(\left[0, T\left[; L^{2 \sigma+2}\left(\mathbb{R}^{N}\right)\right) \cap L^{\infty}\left(\left[0, T\left[; L^{2}\left(\mathbb{R}^{N}\right)\right)\right.\right.\right.\right.} \\
& \quad \leq C\left(\left|E_{\alpha}\right|_{L^{\infty}\left(\left[0, T\left[; H^{1}\right)\right.\right.}^{2 \sigma}+|E|_{L^{\infty}\left(\left[0, T\left[; H^{1}\right)\right.\right.}^{2 \sigma}\right) T^{\frac{1}{q^{\prime}}-\frac{1}{q}}\left|D_{\alpha}+E_{\alpha \perp}\right|_{L^{q}\left(\left[0, T\left[; L^{2 \sigma+2}\right)\right.\right.} .
\end{aligned}
$$

As previously, at this step, we can conclude that the left-hand side of (4.7) goes to zero. It remains to be shown that

As,

$$
\nabla D_{\alpha} \rightarrow 0 \text { in } L^{q}\left(\left[0, T\left[; L^{2 \sigma+2}\left(\mathbb{R}^{N}\right)\right) \cap L^{\infty}\left(\left[0, T\left[; L^{2}\left(\mathbb{R}^{N}\right)\right) \forall T<T^{*} .\right.\right.\right.\right.
$$

$$
\nabla D_{\alpha}(t)=\int_{0}^{t} S_{\|}(t-s) \nabla \mathcal{P}_{\|}\left(\left|E_{\alpha \|}+E_{\alpha \perp}\right|^{2 \sigma}\left(E_{\alpha \|}+E_{\alpha \perp}\right)-|E|^{2 \sigma} E\right)(s) \mathrm{d} s,
$$

applying the proposition 2.1 , we have

$$
\left|\nabla D_{\alpha}\right|_{L^{q}\left(\left[0, T\left[; L^{2 \sigma+2}\left(\mathbb{R}^{N}\right)\right) \cap L^{\infty}\left(\left[0, T\left[; L^{2}\left(\mathbb{R}^{N}\right)\right)\right.\right.\right.\right.} \leq C\left|\nabla\left(\left|E_{\alpha}\right|^{2 \sigma} E_{\alpha}-|E|^{2 \sigma} E\right)\right|_{L^{q^{\prime}}\left(\left[0, T\left[; L^{\left.(2 \sigma+2)^{\prime}\left(\mathbb{R}^{N}\right)\right)}\right.\right.\right.} .
$$

The right-hand side is treated as in the previous section to establish the Lemma 3.4. We recall these estimates.

$$
\begin{aligned}
& \left|\nabla\left(\left|E_{\alpha}\right|^{2 \sigma} E_{\alpha}-|E|^{2 \sigma} E\right)\right|_{L^{q^{\prime}}\left(\left[0, T\left[; L^{\left.(2 \sigma+2)^{\prime}\right)}\right.\right.\right.} \leq c\left|E_{\alpha}\right|_{L^{\infty}\left(\left[0, T\left[; L^{2 \sigma+2}\right)\right.\right.}^{2 \sigma}\left|\nabla\left(E_{\alpha}-E\right)\right|_{L^{q^{\prime}\left(\left[0, T\left[; L^{2 \sigma+2}\right)\right.\right.}} \\
& \quad+c\left(\left|E_{\alpha}\right|_{L^{\infty}\left(\left[0, T\left[; L^{2 \sigma+2}\right)\right.\right.}^{2 \sigma-1}+|E|_{L^{\infty}\left(\left[0, T\left[; L^{2 \sigma+2}\right)\right.\right.}^{2 \sigma-1}\right)|\nabla E|_{L^{q^{\prime}}\left(\left[0, T\left[; L^{2 \sigma+2}\right)\right.\right.}\left|E_{\alpha}-E\right|_{L^{\infty}\left(\left[0, T\left[; L^{2 \sigma+2}\right)\right.\right.} .
\end{aligned}
$$

As $H^{1}\left(\mathbb{R}^{N}\right) \subset L^{2 \sigma+2}\left(\mathbb{R}^{N}\right)$ (since $\left.\sigma<\frac{2}{N-2}\right)$, and from Hölder inequality, we obtain

$$
\begin{aligned}
& \left|\nabla D_{\alpha}\right|_{L^{q}\left(\left[0, T\left[; L^{2 \sigma+2}\left(\mathbb{R}^{N}\right)\right) \cap L^{\infty}\left(\left[0, T\left[; L^{2}\left(\mathbb{R}^{N}\right)\right)\right.\right.\right.\right.} \leq \\
& \qquad\left(\left|E_{\alpha}\right|_{L^{\infty}\left(\left[0, T\left[; H^{1}\left(\mathbb{R}^{N}\right)\right)\right.\right.}^{2 \sigma}+\left(\left|E_{\alpha}\right|_{L^{\infty}\left(\left[0, T\left[; H^{1}\left(\mathbb{R}^{N}\right)\right)\right.\right.}^{2 \sigma-1}+|E|_{L^{\infty}\left(\left[0, T\left[; H^{1}\left(\mathbb{R}^{N}\right)\right)\right.\right.}^{2 \sigma-1}\right)|\nabla E|_{L^{q}\left(\left[0, T\left[; L^{2 \sigma+2}\left(\mathbb{R}^{N}\right)\right)\right)\right.}\right) \\
& \times T^{\frac{1}{q^{\prime}}-\frac{1}{q} \mid}\left|E_{\alpha}-E\right|_{L^{\infty}\left(\left[0, T\left[; H^{1}\left(\mathbb{R}^{N}\right)\right)\right.\right.} .
\end{aligned}
$$


As $q^{\prime}<q$, if we choose $T$ small enough (4.7) and (4.8) ensure that

$$
\left|D_{\alpha}\right|_{W^{1, q}\left(\left[0, T\left[; L^{2 \sigma+2}\left(\mathbb{R}^{N}\right)\right) \cap L^{\infty}\left(\left[0, T\left[; H^{1}\left(\mathbb{R}^{N}\right)\right)\right.\right.\right.\right.} \leq \frac{1}{2}\left|D_{\alpha}+E_{\alpha \perp}\right|_{L^{q}\left(\left[0, T\left[; L^{2 \sigma+2}\left(\mathbb{R}^{N}\right)\right) \cap L^{\infty}\left(\left[0, T\left[; H^{1}\left(\mathbb{R}^{N}\right)\right)\right.\right.\right.\right.} .
$$

That is,

$$
\left|D_{\alpha}\right|_{W^{1, q}\left(\left[0, T\left[; L^{2 \sigma+2}\left(\mathbb{R}^{N}\right)\right) \cap L^{\infty}\left(\left[0, T\left[; H^{1}\left(\mathbb{R}^{N}\right)\right)\right.\right.\right.\right.} \leq\left|E_{\alpha \perp}\right|_{L^{q}\left(\left[0, T\left[; L^{2 \sigma+2}\left(\mathbb{R}^{N}\right)\right) \cap L^{\infty}\left(\left[0, T\left[; H^{1}\left(\mathbb{R}^{N}\right)\right)\right.\right.\right.\right.} .
$$

We then have shown that $D_{\alpha}$ goes to zero when $\alpha$ goes to infinity in

$$
W^{1, q}\left(\left[0, T\left[; L^{2 \sigma+2}\left(\mathbb{R}^{N}\right)\right) \cap L^{\infty}\left(\left[0, T\left[; H^{1}\left(\mathbb{R}^{N}\right)\right)\right.\right.\right.\right.
$$

as soon as $T$ is small enough. In fact, we have the same results for all $T<T^{*}$. Assume that there exists $T_{m}<T^{*}$ such that

$$
\begin{aligned}
& D_{\alpha} \rightarrow 0 \text { in } W^{1, q}\left(\left[0, T\left[; L^{2 \sigma+2}\left(\mathbb{R}^{N}\right)\right) \cap L^{\infty}\left(\left[0, T\left[; H^{1}\left(\mathbb{R}^{N}\right)\right) \forall T<T_{m}\right.\right.\right.\right. \\
& D_{\alpha} \nrightarrow 0 \text { in } W^{1, q}\left(\left[0, T_{m}+\eta\left[; L^{2 \sigma+2}\left(\mathbb{R}^{N}\right)\right) \cap L^{\infty}\left(\left[0, T_{m}+\eta\left[; H^{1}\left(\mathbb{R}^{N}\right)\right) \forall \eta>0 .\right.\right.\right.\right.
\end{aligned}
$$

As previously, we show that there exits $\delta>0\left(\delta<T^{*}-T_{m}\right)$ such that

$$
\left|D_{\alpha}\right|_{W^{1, q}}\left(\left[T, T+\delta\left[; L^{2 \sigma+2}\left(\mathbb{R}^{N}\right)\right) \cap L^{\infty}\left(\left[T, T+\delta\left[; H^{1}\left(\mathbb{R}^{N}\right)\right)\right.\right.\right.\right.
$$

$$
\leq C\left|D_{\alpha}(T)\right|_{H^{1}\left(\mathbb{R}^{N}\right)}+\left|E_{\alpha \perp}\right|_{L^{\infty}\left(\left[T, T+\delta\left[; H^{1}\left(\mathbb{R}^{N}\right)\right) \cap L^{q}\left(\left[T, T+\delta\left[; L^{2 \sigma+2}\left(\mathbb{R}^{N}\right)\right)\right.\right.\right.\right.} .
$$

By virtue of the first part of (4.9), we have

$$
D_{\alpha}(T) \rightarrow 0 \text { in } H^{1}\left(\mathbb{R}^{N}\right),\left(T<T_{m}\right)
$$

Also,

$$
E_{\alpha \perp} \rightarrow 0 \text { in } L^{\infty}\left(\left[T, T+\delta\left[; H^{1}\left(\mathbb{R}^{N}\right)\right) \cap L^{q}\left(\left[T, T+\delta\left[; L^{2 \sigma+2}\left(\mathbb{R}^{N}\right)\right) .\right.\right.\right.\right.
$$

Let us choose $T=T_{m}-\frac{\delta}{2}$, then the second relation of (4.9) is false. The Theorem 4.2 is so proved.

The author wish to thank the professors T. Colin and P. Fabrie, for their helpful remarks and their suggestions.

\section{REFERENCES}

[1] L. Bergé and T. Colin, A singular perturbation problem for an envelope equation in plasma physics. Physica D 84 (1995) $437-459$

[2] T. Colin, On the Cauchy problem for a nonlocal, nonlinear Schrödinger equation occurring in plasma Physics. Differential and Integral Equations 6 (1993) 1431-1450.

[3] R.O. Dendy, Plasma dynamics. Oxford University Press, New York (1990).

[4] J. Ginibre and G. Velo, On a class of nonlinear Schrödinger equations. Parts I, II. J. Funct. Anal. 32 (1979) 1-32, 33-71; Part III Ann. Inst. H. Poincaré A 28 (1978) 287-316.

[5] J. Ginibre and G. Velo, The global Cauchy problem for the nonlinear Schrödinger equation revisited. Ann. Inst. H. Poincaré Anal Non Linéaire 2 (1985) 309-402.

[6] E.M. Stein, Singular Integrals and Differentiability properties of Functions. Princeton University Press, Princeton, New Jersey (1970). 